\title{
GREEN'S FUNCTIONS, HARMONIC FUNCTIONS, AND VOLUME COMPARISON
}

\author{
PETER LI \& LUEN-FAI TAM
}

\section{Introduction}

Let $M^{n}$ be a complete, noncompact, $n$-dimensional manifold without boundary, and let $p \in M$ be a fixed point. In this paper we will study some problems in function theory on a class of manifolds $M$ with Ricci curvature satisfying $\operatorname{Ric}_{M}(x) \geq-(n-1) K(1+r(x))^{-2}$, for some constant $K \geq 0$, where $r(x)$ is the distance from $x$ to the fixed point $p$. Our first aim is to give some sufficient conditions for a manifold $M$ in this class to admit a positive Green's function. The problem has a long history. In 1975, Cheng-Yau [10] first provided a necessary condition involving only the volume growth for a complete manifold to admit a positive Green's function. A sharp necessary condition was later proved by Varopoulos in [34], which states that if a complete manifold $M$ has a positive Green's function, then

$$
\int_{1}^{\infty} \frac{t}{V_{p}(t)} d t<\infty
$$

where $V_{p}(t)$ is the volume of the geodesic ball of radius $t$ with center at $p$. However, condition (0.1) is far from sufficient and a counterexample was given in [34]. This sharp necessary condition of Varopoulos was also later proved in [22] and [13] by using different arguments. The first major result for the sufficiency was due to Varopoulos [31] and Li and Yau [25]. Using the estimates for the heat kernel, they proved that if $M$ has nonnegative Ricci curvature everywhere, and $(0.1)$ is satisfied, then $M$ will have a positive Green's function. Furthermore, Li and Yau [25] proved that if this is the case, then for all $x \neq y$ in $M$, the minimal positive Green's function must satisfy

$$
C^{-1} \int_{r(x, y)}^{\infty} \frac{t}{V_{x}(t)} d t \leq G(x, y) \leq C \int_{r(x, y)}^{\infty} \frac{t}{V_{x}(t)} d t,
$$

Received November 12, 1993. The authors were partially supported by NSF grant \#DMS9300422. 
for some constant $C>0$ depending only on the dimension of $M$. A few years later, it was proved in [20] that if the sectional curvature of $M$ is nonnegative outside a compact set, then condition $(0.1)$ is also sufficient for the existence of a positive Green's function. Moreover, the estimate (0.2) is still valid at each end of large volume growth with some obvious modifications. The constant $C$ depends on the manifold and also on $x$. The results in [20] in one sense are generalizations of the results in [31] and [25], because there is no curvature restriction on a compact set. In another sense the results are more restrictive since the assumption is on the sectional curvature rather than the Ricci curvature. The results in [20] were generalized by Kasue [16] to manifolds with asymptotically nonnegative sectional curvature. Recall that a complete noncompact manifold $M$ is said to have asymptotically nonnegative sectional curvature if there is a point $p \in M$ and a continuous nonincreasing function $\lambda:[0, \infty) \rightarrow[0, \infty)$ such that $\int_{0}^{\infty} r \lambda(r) d r<\infty$ and the sectional curvature of $M$ at $x$ satisfies $K_{M}(x) \geq-\lambda(r(x))$, where $r(x)$ is the distance from the point $x$ to $p$. We would like to point out that the method in [20] is quite different from the method in [25]. Up to this point, there is not much progress in the problem of finding sufficient conditions for existence of positive Green's function if we only assume that the Ricci curvature of the manifold is nonnegative outside a compact set, or more generally, that the Ricci curvature satisfies $\operatorname{Ric}_{M}(x) \geq-(n-1) K(1+r(x))^{-2}$. In $\S 1$, we will prove that under this curvature assumption, and if in addition the manifold satisfies a volume comparison condition (VC) (see the definition in $\S 1)$, then condition $(0.1)$ is sufficient to guarantee the existence of a positive Green's function. We should remark that condition (VC) is satisfied by a manifold with nonnegative Ricci curvature everywhere and by each end of a manifold with asymptotically nonnegative sectional curvature; see $\S 3$. Therefore this result can be considered as a generalization and a unified treatment of those in [25], [31], [20], and [16].

In $\S 2$, we will give some basic estimates for the positive Green's function for the class of the manifolds which we consider. They include the estimates for the positive Green's function on a complete noncompact manifold with nonnegative Ricci curvature obtained in [25], and on a complete noncompact manifold with nonnegative sectional curvature outside a compact set in [20] and the generalization in [16]. These estimates will be useful in the study of various spaces of harmonic functions. It was discovered by the authors in [20] and [24], that in order to study the interplay between the geometry and the harmonic functions on a manifold, it is important to study symmetric Green's functions which may be unbounded 
at infinity. Note that in [21] the authors constructed a symmetric Green's function (which might not be positive) on any complete noncompact manifold using compact exhaustion. This kind of Green's functions turn out to be quite useful; see [24]. Therefore, in $\S 2$, we also give estimates for the Green's function constructed in this manner for manifolds satisfying the curvature and the volume comparison assumptions mentioned above.

In $\S 3$, we will apply the results in $\S 1$ and $\S 2$ to give new proofs of the existing estimates for the Green's function given by [25], [31], [20], and [16]. We will also derive some new estimates for complete noncompact manifolds with nonnegative Ricci curvature and small volume growth.

In $\S 4$, we will apply the results in $\S 1$ and $\S 2$ to study spaces of harmonic functions on complete noncompact manifolds with nonnegative Ricci curvature outside a compact set. Using the terminology of [24], we say that a complete noncompact manifold is nonparabolic if it supports a positive Green's function, otherwise, we say the manifold is parabolic. On the other hand, if $M$ is a complete noncompact manifold, and $D$ is a compact set, then an unbounded component of $M \backslash D$ is called an end of $M$ with respect of $D$. If the number of ends of $M$ with respect to any compact set is uniformly bounded above by an integer, then we say that $M$ has finitely many ends. In this case, there is an $R>0$ and an interger $k \geq 1$, such that if $\Omega$ is any bounded domain containing $B_{p}(R)$, then $M \backslash \bar{\Omega}$ has exactly $k$ unbounded components, and we will also denote an unbounded component of $M \backslash \bar{\Omega}$ for a fixed but sufficiently large bounded domain $\Omega$ to be an end. Given an end $E$ of $M$ with respect to some compact set, we say that it is a nonparabolic end if it supports a positive Green's function which satisfies the Neumann boundary condition on $\partial E$. Otherwise, $E$ is said to be a parabolic end. This definition of parabolicity for an end is equivalent to those given in [24]. This fact will be discussed in more detail in $\S 1$. Now let $p$ be a point in $M$, let $s(r)$ be the number of parabolic ends, and let $l(r)$ be the number of nonparabolic ends of $M$ with respect to $B_{p}(r)$, the geodesic ball of radius $r$ with center at $p$. It is easy to see that $s(r)$ and $l(r)$ are nondecreasing in $r$. Let $s(r) \rightarrow s$ and $l(r) \rightarrow l$ as $r \rightarrow \infty$, where $s$ and $l$ may be infinity. It was proved in [24] that

$$
s+l \leq \operatorname{dim} \mathscr{H}^{0}(M) \text {. }
$$

If we further assume that $l \geq 1$, then

$$
s+l \leq \operatorname{dim} \mathscr{H}^{+}(M)
$$

and

$$
l \leq \operatorname{dim} \mathscr{H}_{D}^{\infty}(M)
$$


where $\mathscr{H}^{0}(M)$ is the vector space generated by those harmonic functions which are bounded on one side at each end with respect to some compact set, $\mathscr{H}^{+}(M)$ is the vector space generated by positive harmonic functions, and $\mathscr{H}_{D}^{\infty}(M)$ is the space of bounded harmonic functions with finite Dirichlet integral. We should remark that one does not need any curvature assumption in proving these results. On the other hand, if $M$ has nonnegative Ricci curvature, and $l \geq 1$, then (0.4) and (0.5) become equalities. In fact, by the splitting theorem of Cheeger and Gromoll, if $l \geq 1$, then $l=1$, and by a theorem of Yau [35], $\mathscr{H}^{+}(M)=\mathscr{H}_{D}^{\infty}(M)=$ \{constant functions\}. In general, if $M$ is a connected sum of complete noncompact manifolds with nonnegative Ricci curvature, then $(0.3)-(0.5)$ also become equalities; see [11] and [30]. If the sectional curvature is nonnegative outside a compact set, or more generally, if the manifold has asymptotically nonnegative sectional curvature, then by [20] and [16], we have equalities for (0.3)-(0.5) again. Using the estimates of the Green's function in $\S 2$, we give a partial result for the case where the Ricci curvature is nonnegative outside a compact set under the additional assumption that the manifold also satisfies the volume comparison condition (VC) in $\S 1$. We will show in $\S 4$ that the inequality $(0.5)$ is actually an equality in this case.

In $\S 5$, we will discuss the volume comparison condition (VC). As we mentioned before, the condition is satisfied by a complete noncompact manifold with nonnegative Ricci curvature, or by each end of a manifold with asymptotically nonnegative sectional curvature. We will prove that in a certain sense, the volume comparison condition (VC) will also be satisfied by a manifold whose Ricci curvature is almost nonnegative everywhere. The results in this section are purely Riemannian geometric and are interesting in their own rights.

In $\S 6$, we will consider complete noncompact manifolds with nonnegative Ricci curvature outside a compact set and have finite first Betti number. We will prove that (VC) is essentially satisfied by each end of such a manifold. Furthermore, the results in $\S 1$ are still true, and the results in [20] concerning positive harmonic functions can be extended to this kind of manifolds.

Many results can be generalized easily to manifolds which are quasiisometric to the manifolds we discussed, especially, the results in $\S \S 1,2$, 3 , and 6 . We will point that out in due course.

The authors would like to thank Kwok-keung Au for some useful discussion. Part of the research was done while the second author was visiting the Chinese University of Hong Kong. 


\section{Existence of positive Green's function}

Let $M^{n}$ be a complete, noncompact, connected manifold of dimension $n$. Let $D$ be a compact subset of $M$, and $E$ be an end of $M$ with respect to $D$.

Definition 1.1. $E$ is a nonparabolic end if there exists a positive Green's function on $E$ which satisfies the Neumann boundary condition on $\partial E$. Otherwise, $E$ is said to be a parabolic end.

More precisely, $E$ is a nonparabolic end if $\lim _{R \rightarrow \infty} G_{R}^{N}(x, y)$ exists for all $x \neq y$ where $G_{R}^{N}$ is the positive Green's function on $E \cap B_{p}(R)$ for a fixed point $p$ with mixed boundary conditions $\frac{\partial}{\partial \nu_{y}} G_{R}^{N}(x, y)$ on $\partial E$ and $G_{R}^{N}(x, y)=0$ for $y \in \partial B_{p}(R) \cap E$.

Proposition 1.2. The following statements are equivalent:

(1) E is a nonparabolic end.

(2) There exists a nonconstant positive superharmonic function defined on $E$ such that its infimum is achieved at infinity of $E$.

(3) Any complete manifold $M$ without boundary which is obtained by smoothly gluing two identical copies of $E$ togther is nonparabolic.

Proof. In [24], we used (2) to define nonparabolicity of an end. We showed that this is equivalent to the fact that $E$ is the only end of a complete nonparabolic manifold with boundary. However, this description is misleading because such a manifold might not exist. It only exists if $\partial E$ bounds an $n$-manifold. This prompts us to use (3) as a substitute for an alternate description. The argument in [24] applies to this without much modification. The fact that (1) is equivalent to (2) is new and the argument is rather simple. To see that (1) implies (2), we observe that the positive Neumann Green's function is a positive superharmonic function. The Hopf boundary point lemma asserts that if the infimum is achieved on $\partial E$, then the outward normal derivative must be negative. This contradicts the Neumann boundary condition.

The fact that (2) implies (1) follows from the construction of Green's function in [21] which can be modified for the construction of Neumann Green's function. The positive superharmonic function from (2) simply acts as a barrier as described in [21].

Let $p$ be a fixed point in $M$. For the remaining part of this section, we will assume that the Ricci curvature of $M$ satisfies

$$
\operatorname{Ric}(x) \geq \frac{-(n-1) K}{(1+r(x))^{2}}
$$

for some constant $K \geq 0$, where $r(x)$ is the distance of $x$ from $p$. We 
introduce the following volume comparison condition:

(VC) There exists a constant $\zeta>0$ such that for all $r$ and all $x \in$ $\partial B_{p}(r), V_{p}(r) \leq \zeta V_{x}(r / 2)$, where $V_{x}(r)$ denotes the volume of the geodesic ball $B_{x}(r)$ of radius $r$ with center at $x$. In general, let $M$ be a complete noncompact manifold, and let $E$ be an end of $M$ with respect to some compact set. Let us denote the volume of the set $B_{p}(r) \cap E$ by $V_{p, E}(r)$. $E$ is said to satisfy condition (VC) if there exists a constant $\zeta>0$ such that for all $r$ and all $x \in \partial B_{p}(r) \cap E, V_{p, E}(r) \leq \zeta V_{x, E}\left(\frac{r}{2}\right)$. Let us first point out some facts concerning volume comparison.

Lemma 1.3. Let $M$ be a complete noncompact manifold of dimension $n$, and let $p \in M$ be a fixed point. Suppose the Ricci curvature of $M$ satisfies $\operatorname{Ric}(x) \geq \frac{-(n-1) K}{(1+r(x))^{2}}$ for some nonnegative constant $K$, where $r(x)$ is the distance of $x$ from $p$. Given any $0<\alpha<1$ there is a constant $C=C(p, K, \alpha, n)$ depending only on $p, K, \alpha, n$ such that

$$
V_{p}(r) \leq C(K, \alpha, n) V_{p}(\alpha r)
$$

and

$$
V_{x}(r / 2) \leq C(K, \alpha, n) V_{x}(\alpha r / 2)
$$

for all $x \in \partial B_{p}(r)$.

Proof. Let $\lambda=-\frac{K}{(1+t)^{2}}$, for $t \geq 0$. Let $g$ be the solution of the linear equation

$$
g^{\prime \prime}=\lambda g
$$

with initial condition $g(0)=0$ and $g^{\prime}(0)=1$. Then $g>0$ and $g^{\prime}>1$ for all $t>0$, and

$$
g(t)=\frac{1}{\beta_{1}-\beta_{2}}\left((1+t)^{\beta_{1}}-(1+t)^{\beta_{2}}\right),
$$

where $\beta_{1}=\frac{1}{2}(1+\sqrt{1+4 K})$ and $\beta_{2}=\frac{1}{2}(1-\sqrt{1+4 K})$, for some constant $A$ and $B$. By a standard argument (see [8])

$$
\frac{V_{p}(r)}{V_{p}(\alpha r)} \leq \frac{\int_{0}^{R}\left((1+t)^{\beta_{1}}-(1+t)^{\beta_{2}}\right)^{n-1} d t}{\int_{0}^{\alpha R}\left((1+t)^{\beta_{1}}-(1+t)^{\beta_{2}}\right)^{n-1} d t} .
$$

It is easy to see that the right side is bounded by a constant depending only on $K, \alpha$, and $n$. One can prove the second statement similarly.

Lemma 1.4. Let $M^{n}$ be a complete noncompact manifold as in Lemma 1.3 , such that $M$ satisfies the volume comparison condition (VC) for some $\zeta$ and some $p \in M$. Then for all $r>0$, and $0<\alpha \leq \frac{1}{4}, B_{p}(2 r) \backslash \overline{B_{p}(r)}$ can be covered by $k$ geodesic balls of radius $\frac{\alpha}{2} r$ with centers in $B_{p}(2 r) \backslash \overline{B_{p}(r)}$, 
where $k$ can be bounded by a constant depending only on $n, \zeta, K$, and $\alpha$.

Proof. Let $k$ be the maximal number of disjoint geodesic balls of radius $\frac{\alpha}{2} r$ with centers $x_{1}, \cdots, x_{k}$ in $B_{p}(2 r) \backslash \overline{B_{p}(r)}$. Then $\left\{B_{x_{i}}(\alpha r)\right\}_{i=1}^{k}$ will cover $B_{p}(2 r) \backslash \overline{B_{p}(r)}$. By the condition (VC) and Lemma 1.3,

$$
k V_{p}(3 r) \leq C_{1} \sum_{i=1}^{k} V_{x_{i}}\left(\frac{\alpha}{2} r\right) \leq C_{1} V_{p}(3 r),
$$

for some constant $C_{1}$ depending only on $n, \zeta, K$, and $\alpha$. This implies that $k \leq C_{1}$.

Note that, according to a theorem of Liu [26], the ball covering property in Lemma 1.4 is true without the assumption that $M$ satisfies condition (VC), provided that the Ricci curvature of $M$ is nonnegative outside a compact set. It is still an open question whether each end with respect to a large enough compact set of a complete noncompact manifold with nonnegative Ricci curvature outside a compact set will satisfy (VC). We will discuss this problem in $\S 5$.

We will use the following gradient estimate of Cheng and Yau [10] for positive harmonic functions defined on a geodesic ball.

Lemma 1.5. Let $M^{n}$ be a complete noncompact manifold, and let $x_{0} \in$ $M$, and $R>0$. Suppose the Ricci curvature on $B_{x_{0}}(R)$ is bounded below by $-(n-1) K$, for some constant $K \geq 0$. If $f$ is a positive harmonic function on $B_{x_{0}}(R)$, then

$$
\sup _{x \in B_{x_{0}}(R)}\left(R-r_{0}(x)\right)|\nabla \log f| \leq C(1+\sqrt{K} R),
$$

where $r_{0}(x)$ is the distance from $x$ to $x_{0}$, and $C>0$ is a constant depending only on $n$.

By integrating along a minimal geodesic from a point $x \in B_{x_{0}}\left(\frac{R}{2}\right)$ to $x_{0}$ in the above inequality, we conclude that there exists a constant $C>0$ depending only on $n$ such that

$$
\exp (-C(1+\sqrt{K} R)) \leq \frac{f(x)}{f\left(x_{0}\right)} \leq \exp (C(1+\sqrt{K} R)),
$$

for all positive harmonic functions $f$ on $B_{x_{0}}(R)$. The next lemma is due to Li and Schoen [19, Theorem 1.2], and was generalized to the following form in [23, Theorem 1.1]; see also [28].

Lemma 1.6. Let $M^{n}$ be a complete noncompact manifold. Let $x \in M$, and let $f$ be a nonnegative function satisfying

$$
\Delta f \geq-\beta f
$$


in $B_{x}(2 R)$ for some $R>0$ and some constant $\beta \geq 0$. Suppose the Ricci curvature of $M$ in $B_{x}(2 R)$ is bounded from below by $-(n-1) K$ for some $K \geq 0$. Then

$$
f(x) \leq \exp \left(C_{2}(1+\sqrt{K} R)\right) \frac{V(K, 2 R)}{R^{n}}\left(\frac{1}{V_{x}(R)} \int_{B_{x}(R)} f(y) d y\right),
$$

for some constant $C_{2}>0$ depending only on $n$ and $\beta$. The notation $V(K, 2 R)$ denotes the volume of the geodesic ball of radius $2 R$ in the model space of constant curvature $-K$.

Lemma 1.7. Let $M$ be a complete noncompact manifold of dimension $n$ and $p \in M$ be a fixed point. Suppose that the Ricci curvature of $M$ satisfies $\operatorname{Ric}(x) \geq \frac{-(n-1) K}{(1+r(x))^{2}}$ for some nonnegative constant $K$, where $r(x)$ is the distance of $x$ from $p$, and that $M$ also satisfies condition (VC) for some $\zeta$. Let $f$ be a harmonic function defined on $M \backslash B_{p}\left(\frac{r}{2}\right)$ for some $r>0$. Then for any $x \in B_{p}(2 r) \backslash \overline{B_{p}(r)}$,

$$
\underset{B_{x}(r / 4)}{\operatorname{osc}} f \leq C_{3}\left(\int_{r}^{2 r} \frac{t}{V_{p}(t)} d t\right)^{1 / 2}\left(\int_{B_{p}(3 r) \backslash B_{p}(r / 2)}|\nabla f|^{2}\right)^{1 / 2}
$$

for some constant $C_{3}$ depending only on $n, \zeta$, and $K$. If, in addition, $x$ is also in $E_{r / 2}$, with $E_{r / 2}$ being an unbounded component of $M \backslash \overline{B_{p}\left(\frac{r}{2}\right)}$, then

$$
\underset{B_{x}(r / 4)}{\operatorname{osc}} f \leq C_{3}\left(\int_{r}^{2 r} \frac{t}{V_{p}(t)} d t\right)^{1 / 2}\left(\int_{E_{r / 2} \cap\left(B_{p}(3 r) \backslash B_{p}(r / 2)\right)}|\nabla f|^{2}\right)^{1 / 2} .
$$

Proof. Let $x \in B_{p}(2 r) \backslash \overline{B_{p}(r)}$. For any $y \in B_{x}\left(\frac{r}{4}\right)$, let $\gamma(t)$ be a minimal geodesic parametrized by the arclength from $x$ to $y$ with length $l$. Then $l \leq \frac{r}{4}$ and for all $0 \leq t \leq l$,

and

$$
B_{\gamma(t)}\left(\frac{1}{12} r(\gamma(t))\right) \subset B_{p}(3 r) \backslash B_{p}\left(\frac{r}{2}\right),
$$

$$
B_{\gamma(t)}\left(\frac{r(\gamma(t))}{6}\right) \subset M \backslash B_{p}\left(\frac{3}{8} r\right) .
$$

By the assumption on the Ricci curvature and the Bochner formula, we have

$$
\Delta|\nabla f|^{2} \geq-\beta r^{-2}|\nabla f|^{2}
$$

on $B_{\gamma(t)}\left(\frac{r(\gamma(t))}{6}\right)$ for some constant $\beta \geq 0$ depending only on $n$ and $K$. Hence by Lemma 1.6,

$$
|\nabla f|^{2}(\gamma(t)) \leq \frac{1}{C_{4}} V_{\gamma(t)}^{-1}\left(\frac{1}{12} r(\gamma(t))\right) \int_{B_{\gamma(t)}(r(\gamma(t)) / 12)}|\nabla f|^{2},
$$


for some constant $C_{4}$ depending only on $n$, and $K$. Since $M$ satisfies (VC), by Lemma 1.3 we have

$$
|\nabla f|^{2}(\gamma(t)) \leq \frac{C_{5}}{V_{p}(2 r)} \int_{B_{p}(3 r) \backslash B_{p}\left(\frac{r}{2}\right)}|\nabla f|^{2},
$$

for all $t$ and some $C_{5}$ depending only on $n, \zeta$, and $K$. Hence

$$
\begin{aligned}
|f(x)-f(y)| & \leq \int_{0}^{l}|\nabla f|(\gamma(t)) d t \leq l \sup _{t}|\nabla f|(\gamma(t)) \\
& \leq l\left(\frac{C_{5}}{V_{p}(2 r)} \int_{B_{p}(3 r) \backslash B_{p}(r / 2)}|\nabla f|^{2}\right)^{1 / 2} \\
& \leq \frac{r}{4}\left(\frac{C_{5}}{V_{p}(2 r)} \int_{B_{p}(3 r) \backslash B_{p}(r / 2)}|\nabla f|^{2}\right)^{1 / 2} \\
& \leq C_{3}\left(\int_{r}^{2 r} \frac{t}{V_{p}(t)} d t\right)^{1 / 2}\left(\int_{B_{p}(3 r) \backslash B_{p}(r / 2)}|\nabla f|^{2}\right)^{1 / 2},
\end{aligned}
$$

for some constant $C_{3}$ depending only on $n, \zeta$, and $K$. If $x \in E_{\frac{r}{2}}$, then $B_{\gamma(t)}\left(\frac{r(\gamma(t))}{12}\right)$ is a subset of $E_{r / 2}$ for all $t$. Hence one can replace $B_{p}(3 r) \backslash B_{p}(r)$ by $E_{r / 2} \cap\left(B_{p}(3 r) \backslash B_{p}(r)\right)$ in the above argument and obtain the second inequality. This completes the proof of the lemma.

In general, a complete noncompact manifold may not have a positive Green's function. However, it was shown in [21] that a symmetric Green's function for the Lapacian can always be constructed by compact exhaustion. More precisely, it was proved that there exist $R_{i} \uparrow \infty$ and $c_{i} \geq 0$ such that

$$
G(x, y)=\lim _{i \rightarrow \infty}\left(G_{i}(x, y)-c_{i}\right)
$$

exists for all $x \neq y$, where $G_{i}(x, y)$ is the positive Green's function of $B_{p}\left(R_{i}\right)$ with Dirichlet boundary value. $G(x, y)$ is a symmetic Green's function. The convergence of $G_{i}(x, y)-c_{i}$ is uniform on a compact subset of $M \backslash\{x\}$ as functions of $y$. If $M$ has a positive Green's function, then one can take $c_{i}=0$ and $G(x, y)$ will be the minimal positive Green's function. Using this setup, let us denote $g_{i}(x)=-G_{i}(p, x)+c_{i}$ and $g(x)=-G(p, x)$. Note that $g_{i}=c_{i}$ on $\partial B_{p}\left(R_{i}\right)$ and $g_{i}(x)<c_{i}$ for all $x \in B_{p}\left(R_{i}\right) \backslash\{p\}$. In this notation, we have the following (also see [22]):

Lemma 1.8. Let $M$ be a complete noncompact manifold. For $r>0$ let $M_{r}$ be the union of the unbounded components of $M \backslash \overline{B_{p}(r)}$. Then for 
all $R>r$,

and

$$
\int_{B_{p}(R) \cap M_{r}}|\nabla g|^{2} \leq 4\left(S(R)-i^{*}(r)\right)
$$

$$
\int_{B_{p}(R) \backslash B_{p}(r)}|\nabla g|^{2} \leq 4(S(R)-i(r)),
$$

where $S(R)=\sup _{\partial B_{p}(R)} g, i^{*}(r)=\inf _{\partial M_{r}} g$ and $i(r)=\inf _{\partial B_{p}(r)} g$.

Proof. Since $g_{i}(p)=-\infty$ and $\left.g_{i}\right|_{\partial B_{p}\left(R_{i}\right)}=c_{i} \geq 0$, by the maximum principle,

$$
\inf _{B_{p}\left(R_{i}\right) \cap M_{r}} g_{i}=\inf _{\partial M_{r}} g_{i}
$$

for $R_{i}>r$. Let us now consider the harmonic function $g_{i}-\inf _{\partial M_{r}} g_{i}$ which is nonnegative on $B_{p}\left(R_{i}\right) \cap M_{r}$. For all $a>0$,

$$
\begin{aligned}
& \int_{B_{p}\left(R_{i}\right) \cap M_{r}} \frac{\left|\nabla g_{i}\right|^{2}}{\left(g_{i}-\inf _{\partial M_{r}} g_{i}+a\right)^{2}} \\
& =-\int_{B_{p}\left(R_{i}\right) \cap M_{r}} \Delta \log \left(g_{i}-\inf _{\partial M_{r}} g_{i}+a\right) \\
& =-\int_{\partial B_{p}\left(R_{i}\right) \cap M_{r}} \frac{1}{g_{i}-\inf _{\partial M_{r}} g_{i}+a} \frac{\partial g_{i}}{\partial r} \\
& \quad+\int_{\partial M_{r}} \frac{1}{g_{i}-\inf _{\partial M_{r}} g_{i}+a} \frac{\partial g_{i}}{\partial r} \\
& \quad \leq \int_{\partial M_{r}} \frac{1}{g_{i}-\inf _{\partial M_{r}} g_{i}+a} \frac{\partial g_{i}}{\partial r}
\end{aligned}
$$

where we have used the fact that $g_{i}=c_{i}$ on $\partial B_{p}\left(R_{i}\right)$ and that $\frac{\partial g_{i}}{\partial r}>0$ on $\partial B_{p}\left(R_{i}\right)$. By setting $R>r$, and taking $i$ sufficiently large so that $R_{i}>R$, we obtain

$$
\int_{B_{p}(R) \cap M_{r}} \frac{\left|\nabla g_{i}\right|^{2}}{g_{i}-\inf _{\partial M_{r}} g_{i}+a^{2}} \leq \int_{\partial M_{r}} \frac{1}{g_{i}-\inf _{\partial M_{r}} g_{i}+a} \frac{\partial g_{i}}{\partial r} .
$$

As $i \rightarrow \infty$, we have

$$
\int_{B_{p}(R) \cap M_{r}} \frac{|\nabla g|^{2}}{\left(g-i^{*}(r)+a\right)^{2}} \leq \int_{\partial M_{r}} \frac{1}{g-i^{*}(r)+a} \frac{\partial g}{\partial r} .
$$

Let $\Omega=\left\{x \in M \mid g(x)-i^{*}(r)<0\right\}$, where we assume that $p \in \Omega$ by convention. Obviously, $\Omega \subset M \backslash \bar{M}_{r}$ and $g(x)-i^{*}(r)>0$ on $M \backslash\left(\overline{M_{r}} \cup \bar{\Omega}\right)$. 
Hence

$$
\begin{aligned}
-\int_{\partial M_{r}} \frac{1}{g-i^{*}(r)+a} \frac{\partial g}{\partial r} & +\int_{\partial \Omega} \frac{1}{g-i^{*}(r)+a} \frac{\partial g}{\partial \nu} \\
& =\int_{M \backslash\left(\overline{M_{r}} \cup \bar{\Omega}\right)}\left|\nabla \log \left(g-i^{*}(r)+a\right)\right|^{2} \geq 0,
\end{aligned}
$$

where $\nu$ is the unit outward normal of $\partial \Omega$. Combining this with (1.1) yields

$$
\int_{B_{p}(R) \cap M_{r}} \frac{|\nabla g|^{2}}{\left(g-i^{*}(r)+a\right)^{2}} \leq \int_{\partial \Omega} \frac{1}{g-i^{*}(r)+a} \frac{\partial g}{\partial \nu} .
$$

Since $g(x)$ is the negative of the Green's function, and $g-i^{*}(r)=0$ on $\partial \Omega$, we have

$$
\int_{B_{p}(R) \cap M_{r}} \frac{|\nabla g|^{2}}{\left(g-i^{*}(r)+a\right)^{2}} \leq \frac{1}{a}
$$

for all $a>0$. Set $a=S(R)-i^{*}(r)>0$ and then the lemma follows from the maximum principle and that

$$
\sup _{B_{p}(R) \cap M_{r}} g \leq S(R) .
$$

The second inequality can be proved in a similar manner.

Theorem 1.9. Let $M^{n}$ be a complete noncompact manifold of dimension $n$, and let $p \in M$ be a fixed point. Suppose that the Ricci curvature of $M$ satisfies $\operatorname{Ric}(x) \geq-\frac{(n-1) K}{(1+r(x))^{2}}$ for some nonnegative constant $K$, where $r(x)$ is the distance of $x$ from $p$. Let us also assume that $M$ satisfies condition (VC) for some $\zeta$. If $G(x, y)$ is a Green's function obtained by compact exhaustion, then there is a constant $C_{4}$ depending only on $n, \zeta$, and $K$ such that for all $r_{0}>0$ and all $x \in M$ with $r(x)>2 r_{0}>0$,

$$
\begin{aligned}
& -G(p, x)+\sup _{\partial B_{p}\left(r_{0}\right)} G(p, \cdot) \\
& \quad \leq C_{4}\left(\int_{r_{0}}^{r(x)} \frac{t}{V_{p}(t)} d t+\sup _{\partial B_{p}\left(r_{0}\right)} G(p, \cdot)-\inf _{\partial B_{p}\left(2 r_{0}\right)} G(p, \cdot)\right) .
\end{aligned}
$$

Moreover, $M$ has a positive Green's function if and only if

$$
\int_{1}^{\infty} \frac{t}{V_{p}(t)} d t<\infty
$$

Proof. Let $r_{0}>0$ and $r>2 r_{0}$. Then there exists $k \geq 1$ so that $2^{k} r_{0}<r \leq 2^{k+1} r_{0}$. Let $g(x)=-G(p, x)$, and for any $r>0$, let $S(r)=\sup _{\partial B_{p}(r)} g$ and $i(r)=\inf _{\partial B_{p}(r)} g$. Let $y \in \partial B_{p}\left(2^{k} r_{0}\right)$ be such that 
$g(y)=\sup _{\partial B_{p}\left(2^{k} r_{0}\right)} g$ and let $\gamma(t)$ be a minimal geodesic from $p$ to $y$ parametrized by arclength. Let $y_{i}=\gamma\left(2^{i} r_{0}\right)$ for $i \geq 1$ so that $y=y_{k}$. By Lemma 1.5 , there exists a constant $C_{3}>0$ depending only on $n, \zeta$, and $K$ such that $\left|g\left(y_{i+1}\right)-g\left(y_{i}\right)\right| \leq 4 C_{3}\left(\int_{2^{i} r_{0}}^{2^{i+1} r_{0}} \frac{t}{V_{p}(t)} d t\right)^{1 / 2}\left(\int_{B_{p}\left(3 \cdot 2^{i} r_{0}\right) \backslash B_{p}\left(2^{i-1} r_{0}\right)}|\nabla g|^{2}\right)^{1 / 2}$, for $1 \leq i<k$. Hence

$$
\begin{aligned}
g(y) & -g\left(\gamma\left(2 r_{0}\right)\right) \\
& \leq 4 C_{3} \sum_{i=1}^{k-1}\left(\int_{2^{i} r_{0}}^{2^{i+1} r_{0}} \frac{t}{V_{p}(t)} d t\right)^{1 / 2}\left(\int_{B_{p}\left(3 \cdot 2^{i} r_{0}\right) \backslash B_{p}\left(2^{i-1} r_{0}\right)}|\nabla g|^{2}\right)^{1 / 2} \\
& \leq 4 C_{3}\left(\sum_{i=1}^{k-1} \int_{2^{i} r_{0}}^{2^{i+1} r_{0}} \frac{t}{V_{p}(t)} d t\right)^{1 / 2}\left(\sum_{i=1}^{k-1} \int_{B_{p}\left(3 \cdot 2^{i} r_{0}\right) \backslash B_{p}\left(2^{i-1} r_{0}\right)}|\nabla g|^{2}\right)^{1 / 2} \\
& \leq 12 C_{3}\left(\int_{2 r_{0}}^{2^{k} r_{0}} \frac{t}{V_{p}(t)} d t\right)^{1 / 2}\left(\int_{B_{p}\left(3 \cdot 2^{k-1} r_{0}\right) \backslash B_{p}\left(r_{0}\right)}|\nabla g|^{2}\right)^{1 / 2} .
\end{aligned}
$$

By the second inequality of Lemma 1.8 ,

$$
\int_{B_{p}\left(3 \cdot 2^{k-1} r_{0}\right) \backslash B_{p}\left(r_{0}\right)}|\nabla g|^{2} \leq 4\left(S\left(3 \cdot 2^{k-1} r_{0}\right)-i\left(r_{0}\right)\right) .
$$

Combining with (1.2) and the fact that $g(y)=S\left(2^{k} r_{0}\right)$, we have

$$
\begin{aligned}
& S\left(2^{k} r_{0}\right)-g\left(\gamma\left(2 r_{0}\right)\right) \\
& \quad \leq 24 C_{3}\left(S\left(3 \cdot 2^{k-1} r_{0}\right)-i\left(r_{0}\right)\right)^{1 / 2}\left(\int_{r_{0}}^{2^{k} r_{0}} \frac{t}{V_{p}(t)} d t\right)^{1 / 2} .
\end{aligned}
$$

By the maximum principle and the fact that $G$ is obtained by compact exhaustion, $g-i\left(r_{0}\right)$ is a positive harmonic function on $M \backslash \overline{B_{p}\left(r_{0}\right)}$. Hence Lemma 1.5 and the curvature assumption imply that

$$
S\left(3 \cdot 2^{k-1} r_{0}\right)-i\left(r_{0}\right) \leq C_{5}\left(S\left(2^{k} r_{0}\right)-i\left(r_{0}\right)\right)
$$

for some constant $C_{5}$ depending only on $n$ and $K$. Using the Hölder inequality, (1.3) and (1.4), we conclude that 


$$
\begin{aligned}
S\left(2^{k} r_{0}\right) & -i\left(r_{0}\right) \\
= & S\left(2^{k} r_{0}\right)-g\left(\gamma\left(2 r_{0}\right)\right)+g\left(\gamma\left(2 r_{0}\right)\right)-i\left(r_{0}\right) \\
\leq & C_{6}\left(S\left(2^{k} r_{0}\right)-i\left(r_{0}\right)\right)^{1 / 2}\left(\int_{r_{0}}^{2^{k} r_{0}} \frac{t}{V_{p}(t)} d t\right)^{1 / 2} \\
& +g\left(\gamma\left(2 r_{0}\right)\right)-i\left(r_{0}\right) \\
\leq & C_{7} \int_{r_{0}}^{2^{k} r_{0}} \frac{t}{V_{p}(t)} d t+\frac{1}{2}\left(S\left(2^{k} r_{0}\right)-i\left(r_{0}\right)\right) \\
& +S\left(2 r_{0}\right)-i\left(r_{0}\right)
\end{aligned}
$$

for some constants $C_{6}, C_{7}>0$ depending only on $n, \zeta$, and $K$. The first part of the theorem follows from Lemma 1.3, the curvature assumption, the facts that $2^{k} r_{0}<r \leq 2^{k+1} r_{0}$ and $S\left(2 r_{0}\right)-i\left(r_{0}\right)>0$. As for the second part of the theorem, it was proved in [34] (see also [22]), that if $M$ has a positive Green's function then $\int_{1}^{\infty} \frac{t}{V_{p}(t)} d t<\infty$. Suppose $\int_{1}^{\infty} \frac{t}{V_{p}(t)} d t<\infty$. Then letting $r_{0}=1$ in $(1.5)$, we see that $G(p, x)$ is bounded from below near infinity, and hence $M$ has a positive Green's function. This completes the proof of the theorem.

Remark 1.10. The statement in Theorem 1.9 concerning the necessary and sufficient condition for the existence of a positive Green's function was proved in [31] for manifolds with nonnegative Ricci curvature everywhere (also see [25] and [18]). Their proofs are based on the estimates of the heat kernel. In this case, the volume comparison condition (VC) is a consequence of the Bishop comparison theorem [3]. The same result was proved for manifolds with nonnegative sectional curvature outside a compact set in [20], and was later generalized to manifolds with asymptotically nonnegative sectional curvature in [16]. We will see in $\S 3$ that manifolds of this kind also satisfy condition (VC). Hence the last part of Theorem 1.9 can be considered as a generalization of all these results.

Remark 1.11. It is well known that the property of existence of positive Green's function is quasi-isometric invariant; see for example [13]. Hence the last conclusion of Theorem 1.9 is still true for a complete noncompact manifold which is quasi-isometric to a manifold satisfying the assumptions of the theorem.

\section{Estimates for Green's functions}

In this section, we will give some upper and lower estimates for a symmetric Green's function obtained by compact exhaustion on a complete 
noncompact manifold $M$ satisfying the assumptions in Lemma 1.5. Since the behavior of the Green's function will be different on different ends with respect to a compact set of a manifold, for the sake of convenience, we will assume that the manifold has only one end. This is to say that for all $r>0$ there is only one unbounded component of $M \backslash \overline{B_{p}(r)}$, which will be denoted by $M_{r}$. This assumption is not very restrictive because of the following result. It was proved in [24] that if the Ricci curvature of $M^{n}$ satisfies $\operatorname{Ric}_{M}(x) \geq-\lambda(r(x))$ where $r(x)$ is the distance from $x$ to a fixed point $p$, and $\lambda:[0, \infty) \rightarrow[0, \infty)$ is a nonincreasing function so that $\int_{0}^{\infty} r^{n-1} \lambda(r) d r<\infty$, then $M$ has only finitely many ends. There are two separate cases to be considered: the case where $M$ admits a positive Green's function, and the case where $M$ does not admit any positive Green's function. Let us first study the case where $M$ has a positive Green's function. We begin with a simple lemma.

Lemma 2.1. Let $X$ be a topological space and let $f: X \rightarrow \mathbb{R}$ be a continuous function. Let $\gamma:[0,1] \rightarrow X$ be a continuous curve which is covered by finitely many nonempty open sets $U_{1}, \cdots, U_{k}$. Denote $\gamma(0)=$ $x$, and $\gamma(1)=y$. Then

$$
|f(x)-f(y)| \leq \sum_{i=1}^{k} \underset{U_{i}}{\operatorname{osc}} f .
$$

Proof. We will prove the lemma by induction on $k$. The lemma is obviously true for $k=1$. Suppose that the lemma is true for $k$, and that $\gamma$ is covered by a family of nonempty open sets $U_{1}, \cdots, U_{k}, U_{k+1}$. Without loss of generality, we may assume that $x=\gamma(0) \in U_{k+1}$. Let $t_{1}$ to be the supremum of $t$ in $[0,1]$ such that $\gamma(t) \in U_{k+1}$. Then $\gamma\left(t_{1}\right)$ is in the closure of $U_{k+1}$. If $t_{1}=1$, then

$$
\begin{aligned}
|f(x)-f(y)| & =\left|f(x)-f\left(\gamma\left(t_{1}\right)\right)\right| \\
& \leq \underset{\bar{U}_{k+1}}{\operatorname{osc}} f=\underset{U_{k+1}}{\operatorname{osc}} f,
\end{aligned}
$$

and we are done. Suppose $t_{1}<1$. Thus by the definition of $t_{1}$ for any $\epsilon>0$ so that $t_{1}+\epsilon<1,\left.\gamma\right|_{\left[t_{1}+\epsilon, 1\right]}$ can be covered by $U_{1}, \cdots, U_{k}$. By the induction hypothesis,

$$
\left|f\left(\gamma\left(t_{1}+\epsilon\right)\right)-f(y)\right| \leq \sum_{i=1}^{k} \underset{U_{i}}{\operatorname{osc}} f .
$$

Since $\left|f(x)-f\left(\gamma\left(t_{1}\right)\right)\right| \leq \operatorname{osc}_{U_{k+1}} f$, the lemma follows by letting $\epsilon \rightarrow 0$ in the above inequality. 
Lemma 2.2. Let $M$ be a complete noncompact manifold of dimension $n$ with only one end, and let $p \in M$ be a fixed point such that that $\int_{1}^{\infty} \frac{t}{V_{p}(t)} d t<\infty$. Suppose that the Ricci curvature of $M$ satisfies $\operatorname{Ric}(x) \geq-\frac{(n-1) K}{(1+r(x))^{2}}$ for some nonnegative constant $K$, where $r(x)$ is the distance of $x$ from $p$. Suppose $M$ also satisfies condition (VC) for some $\zeta$. Let $r>0$ and let $f$ be a harmonic function defined on $M_{r / 2}$ with finite Dirichlet integral, where $M_{r}$ is the unbounded component of $M \backslash \overline{B_{p}(r)}$. If $x$ and $y$ are in $M_{\frac{1}{2}}$ such that $x$ and $y$ can be joined by a curve $\gamma \subset M \backslash \overline{B_{p}(r)}$, then

$$
|f(x)-f(y)| \leq C_{1}\left(\int_{r}^{\infty} \frac{t}{V_{p}(t)} d t\right)^{1 / 2}\left(\int_{M_{r / 2}}|\nabla f|^{2}\right)^{1 / 2}
$$

for some constant $C_{1}>0$ depending only on $n, \zeta$, and $K$.

Proof. Let $x$ and $y$ be two points in $M_{r / 2}$, which can be joined by a continuous curve $\gamma(t), 0 \leq t \leq 1$, in $M \backslash \overline{B_{p}(r)}$ such that $\gamma(0)=x$ and $\gamma(1)=y$. In particular, $x$ and $y$ are both in $M \backslash \overline{B_{p}(r)}$. Let us point out that the points $x$ and $y$ may not be in $M_{r}$, the unbounded component of $M \backslash \overline{B_{p}(r)}$. Let $J \geq 1$ be an integer such that $\gamma \subset M_{r / 2} \cap$ $\left(B_{p}\left(2^{J} r\right) \backslash \overline{B_{p}(r)}\right)$. By the ball covering Lemma 1.4, for all $j \geq 1$, there exist $x_{1}^{(j)}, \cdots, x_{s(j)}^{(j)}$ in $B_{p}\left(2^{j} r\right) \backslash \overline{B_{p}\left(2^{j-1} r\right)}$, such that $B_{p}\left(2^{j} r\right) \backslash \overline{B_{p}\left(2^{j-1} r\right)}$ can be covered by $\left\{B_{i}^{(j)}\right\}_{i=1}^{s(j)}$, where $B_{i}^{(j)}=B_{x_{i}^{(j)}}\left(\frac{1}{4} \cdot 2^{j-1} r\right)$. Moreover, $s(j)$ is bounded by a constant depending only on $n, \zeta$, and $K$. Note that if $B_{i}^{(j)} \cap M_{r / 2} \neq \varnothing$, then $B_{i}^{(j)} \subset M_{r / 2}$. Therefore if we select those $B_{i}^{(j)}$ which have nonempty intersection with $M_{r / 2}$, and still call them $B_{i}^{(j)}$, then $\left.M_{r / 2} \cap \overline{\left(B_{p}\left(2^{j} r\right)\right.} \backslash B_{p}\left(2^{j-1} r\right)\right)$ can be covered by balls $B_{i}^{(j)}, 1 \leq i \leq$ $s(j)$, so that each of the balls is contained in $M_{\frac{r}{2}}$. Moreover, $B_{i}^{(j)} \subset$ $B_{p}\left(3 \cdot 2^{j-1} r\right) \backslash B_{p}\left(2^{j-2} r\right)$. By Lemma 2.1 , since $\gamma$ is covered by $\left\{B_{i}^{(j)}\right\}$, we have

$$
|f(x)-f(y)| \leq \sum_{j=1}^{J} \sum_{i=1}^{s(j)} \underset{B_{i}^{(j)}}{\operatorname{osc}} f
$$

If we denote

$$
M_{r / 2} \cap\left(B_{p}\left(3 \cdot 2^{k-1} r\right) \backslash B_{p}\left(2^{k-2} r\right)\right)
$$

by $M_{r, k}$, then by $(2.1)$, Lemma 1.7 , and the definition of the $B_{i}^{(j)}$, we 
have

$$
\begin{aligned}
|f(x)-f(y)| & \leq C_{2} \sum_{k=1}^{\infty}\left(\int_{2^{k-1},}^{2^{k} r} \frac{t}{V_{p}(t)} d t\right)^{1 / 2}\left(\int_{M_{r, k}}|\nabla f|^{2}\right)^{1 / 2} \\
& \leq C_{2}\left(\sum_{k=1}^{\infty} \int_{2^{k-1} r}^{2^{k} r} \frac{t}{V_{p}(t)} d t\right)^{1 / 2}\left(\sum_{k=1}^{\infty} \int_{M_{r, k}}|\nabla f|^{2}\right)^{1 / 2} \\
& \leq 3 C_{2}\left(\int_{r}^{\infty} \frac{t}{V_{p}(t)} d t\right)^{1 / 2}\left(\int_{M_{r / 2}}|\nabla f|^{2}\right)^{1 / 2}
\end{aligned}
$$

for some constant $C_{2}>0$ depending only on $\zeta, n$, and $K$. Here we have used the fact that $s(j)$ is bounded from above by a constant depending only on $n, \zeta$, and $K$. This completes the proof of the lemma.

The right side of the inequality in Lemma 2.2 involves the Dirichlet integral of $f$ over $M_{r / 2}$ rather than over $M_{r}$. This is not enough to give an estimate for the Green's function. However, if $f$ is also positive, then using Lemma 2.2, one can prove the following:

Lemma 2.3. Let $M$ be as in Lemma 2.2. Let $r>0$, and $f$ be a positive harmonic function on $M_{r / 2}$ with finite Dirichlet integral. Then for any $x$ and $y$ in $M_{r}$, we have

$$
f(x) \leq C_{3}\left(\int_{r}^{\infty} \frac{t}{V_{p}(t)} d t\right)^{1 / 2}\left(\int_{M_{r}}|\nabla f|^{2}\right)^{1 / 2}+C_{4} f(y),
$$

for some constants $C_{3}$ and $C_{4}$ depending only on $n, \zeta$, and $K$.

Proof. Let $x$ and $y$ be in $M_{r}$. Then there is a continuous curve $\gamma(t)$, $0 \leq t \leq 1$, in $M_{r}$ such that $\gamma(0)=x$ and $\gamma(1)=y$. By Lemma 1.4, we can find $x_{1}, \cdots, x_{s}$ in $B_{p}(2 r) \backslash \overline{B_{p}(r)}$ such that $\bigcup_{i=1}^{s} B_{i} \supset B_{p}(2 r) \backslash \overline{B_{p}(r)}$, where $B_{i}=B_{x_{i}}\left(\frac{r}{8}\right)$, and $s$ is bounded by a constant depending only on $n, \zeta$, and $K$. Since $\gamma \subset M_{r}$, if $B_{i} \cap \gamma \neq \varnothing$ then $B_{i} \subset M_{3 r / 4}$. Suppose $\gamma \cap\left(\bigcup_{i=1}^{s} B_{i}\right) \neq \varnothing$, then there is a smallest $t_{1}$ such that $\gamma\left(t_{1}\right) \in \bigcup_{i=1}^{s} \overline{B_{i}}$. We may assume that $\gamma\left(t_{1}\right) \in \overline{B_{1}}$. Let $t_{1}^{\prime} \geq t_{1}$ be the largest number in $[0,1]$ so that $\gamma\left(t_{1}^{\prime}\right) \in \overline{B_{1}}$. Then

(1) $\gamma\left(\left(0, t_{1}\right)\right) \subset M \backslash B_{p}(2 r)$;

(2) $\gamma\left(t_{1}\right)$ and $\gamma\left(t_{1}^{\prime}\right)$ are in $\overline{B_{1}}$; and

(3) either $t_{1}^{\prime}=1$ or $\gamma\left[t_{1}^{\prime}, 1\right] \cap B_{1}=\varnothing$.

If $t_{1}^{\prime}=1$ or $\gamma\left(\left[t_{1}^{\prime}, 1\right]\right) \cap\left(\bigcup_{i=1}^{s} B_{i}\right)=\varnothing$, then we stop. If $t_{1}^{\prime}<1$ and $\gamma\left(\left[t_{1}^{\prime}, 1\right]\right) \cap\left(\bigcup_{i=1}^{s} B_{i}\right) \neq \varnothing$, then by $(3), \gamma\left(\left[t_{1}^{\prime}, 1\right]\right) \cap\left(\bigcup_{i=2}^{s} B_{i}\right) \neq \varnothing$. Arguing 
as before and if necessary renaming the $B_{i}$ 's for $2 \leq i \leq m$, we can find $t_{1}^{\prime} \leq t_{2} \leq t_{2}^{\prime} \leq 1$, such that

(1) $\gamma\left(\left(t_{1}^{\prime}, t_{2}\right)\right) \subset M \backslash B_{p}(2 r)$;

(2) $\gamma\left(t_{2}\right)$ and $\gamma\left(t_{2}^{\prime}\right)$ are in $\overline{B_{2}}$; and

(3) either $t_{2}^{\prime}=1$ or $\gamma\left(\left[t_{2}^{\prime}, 1\right]\right) \cap\left(B_{1} \cup B_{2}\right)=\varnothing$.

If $t_{2}^{\prime}=1$ or $\gamma\left(\left[t_{2}^{\prime}, 1\right]\right) \cap\left(\bigcup_{i=1}^{s} B_{i}\right)=\varnothing$, then we stop. Otherwise we can proceed as before and find $t_{3}$ and $t_{3}^{\prime}$. Continuing in this manner, we can find $0 \leq t_{1} \leq t_{1}^{\prime} \leq t_{2} \leq t_{2}^{\prime} \cdots t_{m} \leq t_{m}^{\prime} \leq 1$, with $m \leq s$ such that

(1) $\gamma\left(\left(0, t_{1}\right) \cup\left(t_{1}^{\prime}, t_{2}\right) \cup \cdots \cup\left(t_{m}^{\prime}, 1\right)\right) \subset M \backslash B_{p}(2 r)$;

(2) $\gamma\left(t_{i}\right)$ and $\gamma\left(t_{i}^{\prime}\right)$ are in $\overline{B_{i}}$, for $1 \leq i \leq m$; and

(3) either $t_{m}^{\prime}=1$ or $\gamma\left(\left[t_{m}^{\prime}, 1\right]\right) \cap\left(B_{1} \cup \cdots \cup B_{s}\right)=\varnothing$.

Since $\gamma\left(t_{i}\right)$ and $\gamma\left(t_{i}^{\prime}\right)$ are in $\overline{B_{i}}$ which is a subset of $\overline{M_{\frac{3}{4} r}}$, by the Harnack inequality derived from the gradient estimate of Lemma 1.5 , the curvature assumption, and the fact that $f$ is positive, there exists a constant $C_{5}>0$ depending only on $n$ and $K$ such that

$$
f\left(\gamma\left(t_{i}\right)\right) \leq C_{5} f\left(\gamma\left(t_{i}^{\prime}\right)\right)
$$

for $1 \leq i \leq m$. Since $\gamma\left(\left(t_{i}^{\prime}, t_{i+1}\right)\right) \subset M \backslash B_{p}(2 r)$ for all $1 \leq i \leq m-1$, and $\gamma \subset M_{r}$, Lemma 2.2 implies that

$$
\begin{aligned}
f\left(\gamma\left(t_{i}^{\prime}\right)\right) & \leq C_{1}\left(\int_{2 r}^{\infty} \frac{t}{V_{p}(t)} d t\right)^{1 / 2}\left(\int_{M_{r}}|\nabla f|^{2}\right)^{1 / 2}+f\left(\gamma\left(t_{i+1}\right)\right) \\
& \leq C_{1}\left(\int_{r}^{\infty} \frac{t}{V_{p}(t)} d t\right)^{1 / 2}\left(\int_{M_{r}}|\nabla f|^{2}\right)^{1 / 2}+f\left(\gamma\left(t_{i+1}\right)\right)
\end{aligned}
$$

for $1 \leq i \leq m-1$, where $C_{1}$ is the constant in Lemma 2.2, which depends only on $n, \zeta$, and $K$. Similarly, if $0<t_{1}$ then

$$
f(x) \leq C_{1}\left(\int_{r}^{\infty} \frac{t}{V_{p}(t)} d t\right)^{1 / 2}\left(\int_{M_{r}}|\nabla f|^{2}\right)^{1 / 2}+f\left(\gamma\left(t_{1}\right)\right)
$$

and if $t_{m}^{\prime}<1$ then

$$
f\left(\gamma\left(t_{m}^{\prime}\right)\right) \leq C_{1}\left(\int_{r}^{\infty} \frac{t}{V_{p}(t)} d t\right)^{1 / 2}\left(\int_{M_{r}}|\nabla f|^{2}\right)^{1 / 2}+f(y) .
$$

Combining (2.2) $-(2.5)$ and using the fact that $m$ is bounded by a constant depending only on $n, \zeta$, and $K$, we thus prove the lemma. 
Theorem 2.4. Let $M$ be as in Lemma 2.2, so that $\int_{1}^{\infty} \frac{t}{V_{p}(t)} d t<\infty$ and $M$ has only one end. Let $G(x, y)$ be the minimal positive Green's function on $M$. Then there is a constant $C_{6}>0$ depending only on $n, \zeta$, and $K$ such that

$$
G(p, x) \leq C_{6} \int_{r}^{\infty} \frac{t}{V_{p}(t)} d t
$$

for all $x \in M_{r}$, where $M_{r}$ is the unbounded component of $M \backslash \overline{B_{p}(r)}$.

Proof. Denote $G(p, x)$ by $f(x)$. Since $f(p)=+\infty$ and $G$ is the limit of Green's functions with Dirichlet boundary value on a compact exhaustion, the maximum principle implies that $\sup _{M_{r}} f=\sup _{\partial M_{r}} f$. It is well known (see for example [24]) that $f$ has finite Dirichlet integral on $M \backslash \overline{B_{p}(r)}$ for all $r>0$. Let $x \in \partial M_{r}$ be such that $f(x)=\sup _{\partial M_{r}} f$. Since $G$ is the minimal positive Green's function, there exists $y_{k} \rightarrow \infty$ such that $f\left(y_{k}\right) \rightarrow 0$ as $k \rightarrow \infty$. Without loss of generality, we may assume $y_{k} \in M_{r}$ for all $k$. By Lemma 2.3,

$$
f(x) \leq C_{7}\left(\int_{r}^{\infty} \frac{t}{V_{p}(t)} d t\right)^{1 / 2}\left(\int_{M_{r}}|\nabla f|^{2}\right)^{1 / 2}+C_{8} f\left(y_{k}\right),
$$

for all $k$, where $C_{7}$ and $C_{8}$ are the contants depending only on $n$, $\zeta$, and $K$. Letting $k \rightarrow \infty$ and using Lemma 1.8 and the fact that $\inf _{\partial B_{p}(R)} f \rightarrow 0$ as $R \rightarrow \infty$, we obtain

$$
\begin{aligned}
f(x) & \leq C_{7}\left(\int_{r}^{\infty} \frac{t}{V_{p}(t)} d t\right)^{1 / 2}\left(\int_{M_{r}}|\nabla f|^{2}\right)^{1 / 2} \\
& \leq 2 C_{7}\left(\int_{r}^{\infty} \frac{t}{V_{p}(t)} d t\right)^{1 / 2}\left(\sup _{\partial M_{r}} f\right)^{1 / 2} \\
& =2 C_{7}\left(\int_{r}^{\infty} \frac{t}{V_{p}(t)} d t\right)^{1 / 2}(f(x))^{1 / 2}
\end{aligned}
$$

The theorem then follows.

By Theorem 2.4, if we let $S^{*}(r)=\sup _{\partial M_{r}} G(p, \cdot)$, then

$$
S^{*}(r) \leq C_{6}\left(\int_{r}^{\infty} \frac{t}{V_{p}(t)} d t\right) .
$$

With some modifications in the proof of Corollary 2.4 in [22], we can obtain a lower bound for $S^{*}(r)$. More precisely, we have 
Proposition 2.5. Let $M$ be a complete noncompact manifold satisfying the same assumptions as in Theorem 2.4. Let $S^{*}(r)=\sup _{\partial M_{r}} G(p, \cdot)$, where $G$ is the minimal positive Green's function on $M$. Then

$$
C_{9} \int_{r}^{\infty} \frac{t}{V_{p}(t)} d t \leq S^{*}(r)
$$

for some constant $C_{9}>0$ depending only on $n, \zeta$, and $K$.

Proof. Let $i(R)=\inf _{\partial B_{p}(R)} G(p, \cdot)$. Then $i(R) \leq S^{*}(r)$ for $R>r$, and $R$ is large enough so that $\partial B_{p}(R) \subset M_{r}$. For $t \geq r$, let $V_{p}^{*}(t)$ be the volume of $\left(B_{p}(t) \cap M_{r}\right) \cup B_{p}(r)$. As in the proof of Corollary 2.4 in [22], using Lemma 1.3 , by considering $-G(p, \cdot)$, we can prove that there exists a constant $C_{10}>0$ depending only on $n, \zeta$, and $K$ such that for $R>r$ sufficiently large

$$
C_{10} \int_{r}^{R} \frac{t}{V_{p}^{*}(t)} d t \leq-i(R)+S^{*}(r) .
$$

By Lemma 1.3, $V_{p}^{*}(t) \leq V_{p}(t)$, and $i(R) \rightarrow 0$ as $R \rightarrow \infty$, the result follows.

Next we will consider the case where $\int_{1}^{\infty} \frac{t}{V_{p}(t)} d t=\infty$. In this case, $M$ does not support any positive Green's function. As mentioned in $\S 1$, it was proved in [21] that there exist $R_{i} \uparrow \infty$ and $c_{i} \geq 0$ such that

$$
G(x, y)=\lim _{i \rightarrow \infty}\left(G_{i}(x, y)-c_{i}\right)
$$

exists for all $x \neq y$, where $G_{i}(x, y)$ is the positive Green's function of $B_{p}\left(R_{i}\right)$ with Dirichlet boundary value. By combining Theorem 1.9, Corollary 2.4 in [22], and Lemma 1.3, we have:

Theorem 2.6. Let $M$ be a complete noncompact manifold of dimension $n$, and let $p \in M$ be a fuxed point. Suppose that the Ricci curvature of $M$ satisfies $\operatorname{Ric}(x) \geq-\frac{(n-1) K}{(1+r(x))^{2}}$ for some nonnegative constant $K$, where $r(x)$ is the distance of $x$ from $p$. Let us also assume that $M$ satisfies condition (VC) for some $\zeta$ and that $\int_{1}^{\infty} \frac{t}{V_{p}(t)} d t=\infty$. If $G(x, y)$ is a Green's function obtained by compact exhaustion, then the following estimates hold:

(1) There is a constant $C_{11}>0$ depending only on $n, \zeta$, and $K$ such that if $r_{0}>0$ and $r(x)>2 r_{0}>0$, then

$$
\begin{aligned}
& -G(p, x)+\sup _{\partial B_{p}\left(r_{0}\right)} G(p, \cdot) \\
& \quad \leq C_{11}\left(\int_{r_{0}}^{r(x)} \frac{t}{V_{p}(t)} d t+\sup _{\partial B_{p}\left(r_{0}\right)} G(p, \cdot)-\inf _{\partial B_{p}\left(2 r_{0}\right)} G(p, \cdot)\right) ;
\end{aligned}
$$


(2) There exists a constant $C_{12}>0$ depending only on $n, \zeta$, and $K$, such that for $R>r>0$,

$$
C_{12} \int_{r}^{R} \frac{t}{V_{p}(t)} d t \leq \sup _{\partial B_{p}(R)}(-G(p, \cdot))+\sup _{\partial B_{p}(r)} G(p, \cdot) .
$$

Remark 2.7. Let $E$ be an end of a complete manifold satisfying (VC). Suppose $M$ is the double of $E$ such that $M$ has exactly two ends and satisfies the curvature assumption in Theorem 2.4. By the proofs of Theorem 2.4 and Proposition 2.5, it is easy to see that the results are still true for $M$. In this case, $M_{r}$ is the union of the two unbounded components of $M \backslash \overline{B_{p}(r)}$.

Remark 2.8. If $M$ is a complete manifold quasi-isometric to a manifold satisfying the assumptions in Theorem 2.4 , then by the estimates of Green's functions on compact domains in terms of capacity in [17] (see also [29]), using the notation in Theorem 2.4, from Theorem 2.4 and the proof of Proposition 2.5 we can deduce that

$$
i^{*}(r) \leq C \int_{r}^{\infty} \frac{t}{V_{p}(t)} d t \leq S^{*}(r)
$$

for some constant $C$, where

$$
i^{*}(r)=\inf _{x \in \partial M_{r}} G(p, x) \text { and } S^{*}(r)=\sup _{x \in \partial M_{r}} G(p, x) .
$$

\section{Applications}

As an application of the results in previous sections, we will give new proofs of the estimates for the minimal positive Green's function on a complete noncompact manifold with nonnegative Ricci curvature obtained in [25]. We will also give some new estimates for the Green's function obtained by compact exhaustion, when the volume growth of $M$ is small so that $M$ does not admit any positive Green's function. We will give new proofs of the estimates, which were obtained in [20], [16], for Green's function on a complete noncompact manifold with asymptotically nonnegative sectional curvature. First let us assume that $M^{n}$ is a complete noncompact manifold with nonnegative Ricci curvature everywhere. Then by the Bishop comparison theorem [3], $M$ satisfies condition (VC) for any $p \in M$ with a constant $\zeta>0$ depending only on $n$. Using the results in $\S \S 1$ and 2, one can prove the following theorem by Li and Yau [25]:

Theorem 3.1. Let $M^{n}$ be a complete noncompact manifold with nonnegative Ricci curvature. Suppose $\int_{1}^{\infty} \frac{t}{V_{x_{0}}(t)}<\infty$, for some point $x_{0}$. Then 
$M$ admits a positive Green's function. Moreover, if $G(x, y)$ is the minimal positive Green's function, then

$$
C(n)^{-1} \int_{r(x, y)}^{\infty} \frac{t}{V_{x}(t)} d t \leq G(x, y) \leq C(n) \int_{r(x, y)}^{\infty} \frac{t}{V_{x}(t)} d t
$$

for some constant $C(n)>0$ depending only on $n$.

Before we prove the theorem, we need the following lemma.

Lemma 3.2. Let $M^{n}$ be as in Theorem 3.1. Let $p \in M, R>0$, and $G_{R}(x, y)$ be the positive Green's function on $B_{p}(R)$ with Dirichlet boundary data. For all $x, y$ in $\partial B_{p}(r)$ with $x \neq y$ and $0<r \leq \frac{R}{5}$, we have

$$
G_{R}(p, x) \leq C_{1} G_{R}(p, y),
$$

for some constant $C_{1}$ depending only on $n$.

Proof. Let $x, y$, and $r$ be as assumed. Let $\gamma$ be a minimal geodesic from $x$ to $y$. By the triangle inequality, it is easy to see that the length of $\gamma$ is not greater than $2 r$ and so $\gamma \subset \overline{B_{p}(3 r)} \subset B_{p}(R)$. In particular, $\operatorname{dist}\left(\gamma, \partial B_{p}(R)\right) \geq r$. Suppose $\gamma \cap B_{p}\left(\frac{r}{3}\right)=\varnothing$. Then by the gradient estimate in Lemma 1.5, we see that (3.1) is true. Suppose that $\gamma \cap B_{p}\left(\frac{r}{3}\right) \neq$ $\varnothing$. Let $\gamma(t) \in B_{p}\left(\frac{r}{3}\right)$. Then

$$
d(x, \gamma(t)) \geq d(p, x)-d(p, \gamma(t)) \geq \frac{2}{3} r .
$$

Similarly, $d(y, \gamma(t)) \geq \frac{2}{3} r$. Therefore

$$
d(x, y) \geq \frac{4}{3} r \text {. }
$$

Let $\alpha(s)$ be a minimal geodesic from $p$ to $x$. Then for all $s$

$$
\begin{aligned}
d(y, \alpha(s)) & \geq d(x, y)-d(x, \alpha(s)) \\
& \geq d(x, y)-d(p, x) \geq \frac{4}{3} r-r \geq \frac{1}{3} r .
\end{aligned}
$$

Hence applying Lemma 1.5 to the positive harmonic function $G_{R}(y, \cdot)$, we have

$$
G_{R}(y, p) \leq C_{2} G_{R}(y, x),
$$

where $C_{2}$ is a constant depending only on $n$. Similarly, one can prove that

$$
G_{R}(x, y) \leq C_{2} G_{R}(x, p) .
$$

By (3.2), and (3.3) and the fact that $G_{R}(x, y)=G_{R}(y, x)$ for all $x$ and $y,(3.1)$ is still true in this case. 
Proof of the theorem. Let us first observe that the integral $\int_{1}^{\infty} \frac{t}{V_{x}(t)} d t$ is finite for some point $x \in M$ implies that the integral is finite for all points $x \in M$. Let $p$ be a fixed point in $M$. Since $G(x, y)=\lim _{R \rightarrow \infty} G_{R}(x, y)$, from (3.1) we see that it is true for all $x \neq y$ in $\partial B_{p}(r)$ if we replace $G_{R}$ by $G$. (Note that (3.1) is obviously true if we assume the theorem is true.) Since $M$ has a positive Green's function, by Theorem 1.9, $\int_{1}^{\infty} \frac{t}{V_{p}(t)} d t<$ $\infty$. The splitting theorem of Cheeger and Gromoll implies that $M$ has only one end. Theorem 3.1 then follows from Lemma 3.2, Theorem 2.4, and Proposition 2.5.

If $M$ is a complete noncompact manifold which is quasi-isometric to a manifold with nonnegative Ricci cuvature satisfying the volume growth condition in Theorem 3.1, then the minimal positive Green's function also satisfies the estimates in the theorem with constants depending on $M$. First noting that Lemma 3.2 is still true by using the Harnack inequality developed in [14] and [28]. The estimates then follows from Lemma 3.2 and Remark 2.8. By the estimate of heat kernels, these estimates for the minimal positive Green's function have been obtained independently by Grigor'yan [14] and Saloff-Coste [28].

The following theorem gives estimates for the Green's function when the volume growth of the manifold is small.

Theorem 3.3. Let $M^{n}$ be a complete noncompact manifold with nonnegative Ricci curvature. Suppose $\int_{1}^{\infty} \frac{t}{V_{x_{0}}(t)}=\infty$, for some point $x_{0}$. Let $G(p, x)$ be a symmetric Green's function obtained by compact exhaustion. Then there exist positive constants $C_{3}$ and $C_{4}$ depending only on $n$ such that for all $r_{0}>0$ and $r>2 r_{0}$, the following hold:

(1) For all $x \neq p$ with $x \in \partial B_{p}(r)$,

$$
\begin{aligned}
& -G(p, x)+\sup _{\partial B_{p}\left(r_{0}\right)} G(p, \cdot) \\
& \quad \leq C_{3}\left(\int_{r_{0}}^{r(x)} \frac{t}{V_{p}(t)} d t+\sup _{\partial B_{p}\left(r_{0}\right)} G(p, \cdot)-\inf _{\partial B_{p}\left(2 r_{0}\right)} G(p, \cdot)\right),
\end{aligned}
$$

where $r(x)=r$ is the distance between $x$ and $p$.

(2) For all $x \in \partial M_{r}$, where $M_{r}$ is the union of the unbounded components of $M \backslash \frac{r}{B_{p}(r)}$,

$$
C_{4} \int_{r_{0}}^{r(x)} \frac{t}{V_{p}(t)} d t \leq-G(p, x)+\sup _{\partial B_{p}\left(r_{0}\right)} G(p, \cdot) .
$$

Proof. The first part is just Theorem 2.6(1), noting that in this case, $M$ satisfies condition (VC) with $\zeta$ depending only on $n$ and has at most 
two ends by [6]. Moreover, by [2], the boundary of each component of $M \backslash \overline{B_{p}(r)}$ is connected. Applying Theorem 2.6(2), the maximum principle, and the fact that $G$ is a Green's function obtained by compact exhaustion, we have

$$
C_{5} \int_{r_{0}}^{r(x)} \frac{t}{V_{p}(t)} d t \leq \sup _{\partial M_{r}}(-G(p, \cdot))+\sup _{\partial B_{p}\left(r_{0}\right)} G(p, \cdot),
$$

for some constant $C_{5}>0$ depending only on $n$. The maximum principle asserts that $-G(p, \cdot)+\sup _{\partial B_{p}\left(r_{0}\right)} G(p, \cdot)$ is positive on $M \backslash \overline{B_{p}\left(r_{0}\right)}$. Suppose $M$ has only one end. Since $r>2 r_{0}$, by Lemmas 1.4 and 1.5 , we conclude that

$$
\sup _{\partial M_{r}}(-G(p, \cdot))+\sup _{\partial B_{p}\left(r_{0}\right)} G(p, \cdot) \leq C_{6}\left(-G(p, x)+\sup _{\partial B_{p}\left(r_{0}\right)} G(p, \cdot)\right)
$$

for some constant $C_{6}>0$ depending only on $n$ for all $x \in \partial M_{r}$. Hence part two of the theorem is true if $M$ has only one end. If $M$ has two ends, then by the splitting theorem of [6], $M$ is isometric to $\mathbb{R} \times M^{\prime}$ where $M^{\prime}$ is a compact manifold with nonnegative Ricci curvature. By a similar argument, the theorem is also true in this case.

Next, we will apply the results in $\S \S 1$ and 2 to give new proofs of the estimates for the Green's functions on certain kinds of manifolds which have been studied by Li and Tam [20] and later generalized by Kasue [16].

Definition 3.4. Let $M$ be a complete noncompact manifold. $M$ is said to have asymtotically nonnegative sectional curvature if there is a point $p$ in $M$ and a continuous, nonnegative, and nonincreasing function $\lambda:[0, \infty) \rightarrow[0, \infty)$ with $\int_{0}^{\infty} t \lambda(t) d t<\infty$, such that the sectional curvature of $M$ satisfies $K_{M}(x) \geq-\lambda(r(x))$, where $r(x)$ is the distance from $x$ to $p$.

Obviously, the definition does not depend on the choice of the point $p$. We should remark that the function $\lambda$ in the definition must satisfy $\lambda(t)=o\left(1 / t^{2}\right)$. Our aim is to prove that if a manifold with asymptotically nonnegative sectional curvature has only one end, then $M$ satisfies condition (VC). Let us first recall some of the properties that these manifolds possess. The following lemma follows from [15], and also from [7] and [20] for the case that $M$ has nonnegative sectional curvature outside a compact set.

Lemma 3.5. Let $M$ be a complete noncompact manifold with asymptotically nonnegative sectional curvature. Then $M$ has only finitely many ends. Furthermore $\partial B_{p}(r) \cap E$ is connected for each end $E$, if $r$ is suffciently large. 
Proof. See [15].

The next lemma was proved in [1].

Lemma 3.6. Let $M$ be as in Lemma 3.5 and $p \in M$. Then there is a constant $C_{7}$ such that for all $r>0$ and $0<\alpha<1, B_{p}(2 r) \backslash B_{p}(r)$ can be covered by $k$ geodesic balls of radius $\alpha r$ with centers in $B_{p}(2 r) \backslash B_{p}(r)$, where $k$ is a constant independent of $r$.

Proof. This is Proposition 1 in §III of [1].

Lemma 3.7. Let $M$ be as in Lemma 3.5 and $p \in M$. Then

$$
\liminf _{r \rightarrow \infty} \frac{V_{p}(2 r)-V_{p}(r)}{V_{p}(r)}>0 .
$$

Proof. Let $A_{p}(r)$ be the area of $\partial B_{p}(r)$. Then Lemma 1 of [16] asserts that

$$
\liminf _{r \rightarrow \infty} \frac{r A_{p}(r)}{V_{p}(r)} \geq 1,
$$

so that there is an $R>0$ such that if $r \geq R$, then

$$
\frac{r A_{p}(r)}{V_{p}(r)} \geq \frac{1}{2}
$$

For $r>R$, we have

$$
\log V_{p}(2 r)-\log V_{p}(r) \geq \frac{1}{2}(\log (2 r)-\log (r)) .
$$

Hence

$$
\frac{V_{p}(2 r)}{V_{p}(r)} \geq \sqrt{2}
$$

and

$$
\frac{V_{p}(2 r)-V_{p}(r)}{V_{p}(r)} \geq \sqrt{2}-1>0,
$$

for all $r>R$. The proof of the lemma is completed.

If $M$ has asymptotically nonnegative sectional curvature, then $M$ has finitely many ends by Lemma 3.5. Let $p \in M$. Then there is $R_{0}>0$ such that the number of unbounded components of $M \backslash \overline{B_{p}(R)}$ is the same for all $R \geq R_{0}$. In the following, an end $M$ is an unbounded component of $M \backslash \overline{B_{p}\left(R_{0}\right)}$.

Proposition 3.8. Let $M$ be a complete noncompact manifold with asymptotically nonnegative sectional curvature. Let $E$ be an end of $M$, and let $M_{E}$ be a complete manifold obtained by gluing two copies of $E$ together. Then $M$ satisfies condition (VC) for some constant $\zeta$. 
Proof. We may assume that $M=M_{E}$. Let $E_{1}$ and $E_{2}$ be the two ends of $M$. Lemma 3.5 asserts that $\partial B_{p}(r) \cap E_{i}$ is connected for $r$ sufficiently large for $i=1,2$. Combining this with Lemma 3.6 and Lemma 3.7, we see that there exists a constant $C_{8}>0$ independent of $r$ such that for all $r>0$ there is $y \in \partial B_{p}(r)$ with

$$
V_{p}(r) \leq C_{8} V_{y}(r / 2) .
$$

We may assume that $y \in E_{1}$. By Lemma 3.5 and Lemma 3.6 again, for any $x \in \partial B_{p}(r) \cap E_{1}$ there is a piecewise smooth curve from $x$ to $y$ in $B_{p}(3 r) \backslash B_{p}\left(\frac{r}{2}\right)$ with length not greater than some constant $C_{9}>0$, which is independent of $x, y$, and $r$. By the curvature assumption, the Ricci curvature in $B_{p}(3 r) \backslash B_{p}\left(\frac{r}{2}\right)$ is bounded below by $-C_{10} r^{-2}$ for some constant $C_{10}>0$ independent of $r$. Hence Lemma 1.3 implies that

$$
V_{y}(r / 2) \leq C_{11} V_{x}(r / 2)
$$

for all $x \in \partial B_{p}(r) \cap E_{1}$ and some constant $C_{11}>0$ independent of $r$, which is sufficiently large. Hence the proposition follows from (3.5), (3.6), and the definition of $M_{E}$.

Combining Theorem 1.9, Theorem 2.4, Proposition 2.5, Theorem 2.6, Lemma 3.5, Proposition 3.8, and the gradient estimate in Lemma 1.5, we can derive the following results in [20] and [16].

Theorem 3.9. Let $M$ be a complete noncompact manifold with asymptotically nonnegative sectional curvature and $p \in M$. Then $M$ admits a positive Green's function if and only if $\int_{1}^{\infty} \frac{t}{V_{p}(t)} d t<\infty$. Furthermore, if $E$ is an end of $M$, and $M_{E}$ is obtained by gluing two copies of $E$ together, then we have the following estimates:

(1) If $\int_{1}^{\infty} \frac{t}{V_{p}(t)} d t<\infty$, and $G(p, x)$ is the minimal positive Green's function of $M$, then there is a constant $C_{12}>0$ such that

$$
C_{12}^{-1} \int_{r(x)}^{\infty} \frac{t}{V_{p}(t)} d t \leq G(p, x) \leq C_{12} \int_{r(x)}^{\infty} \frac{t}{V_{p}(t)} d t
$$

where $r(x)$ is the distance from $x$ to $p$;

(2) If $\int_{1}^{\infty} \frac{t}{V_{p}(t)} d t=\infty$, and $G(p, x)$ is a Green's function obtained by compact exhaustion. Then for all $r_{0}>0$ and $r>2 r_{0}$, there exists a constant $C_{13}>0$ which is independent of $r$ and $r_{0}$ such that

$$
\begin{aligned}
& C_{13}^{-1} \int_{r_{0}}^{r(x)} \frac{t}{V_{p}(t)} d t \leq-G(p, x)+\sup _{\partial B_{p}\left(r_{0}\right)} G(p, \cdot) \\
& \quad \leq C_{13}\left(\int_{r_{0}}^{r(x)} \frac{t}{V_{p}(t)} d t+\sup _{\partial B_{p}\left(r_{0}\right)} G(p, \cdot)-\inf _{\partial B_{p}\left(2 r_{0}\right)} G(p, \cdot)\right) .
\end{aligned}
$$


The above estimates are still true for a manifold which is quasi-isometric to a manifold satisfying the assumptions of Theorem 3.9. In fact, this was proved by Sung [29].

\section{Space of bounded harmonic functions}

Definition 4.1. Let $M$ be a complete noncompact manifold, and let $E$ be an end of $M$ with respect to some compact set. Let us denote the volume of the set $B_{p}(r) \cap E$ by $V_{p, E}(r)$.

(1) $E$ is said to be large, if there exists a point $p \in M$ such that $\int_{1}^{\infty} \frac{t}{V_{p, E}(t)} d t<\infty$

(2) $E$ is said to be small, if $\int_{1}^{\infty} \frac{t}{V_{p, E}(t)} d t=\infty$ for some point $p \in M$.

We say that $M$ has finitely many ends if the number of unbounded components of $M \backslash B_{p}(r)$ is equal to a fixed finite number for all sufficiently large $r$. In this case, when we say an end of $M$ we mean an end of $M$ with respect to $B_{p}(r)$ for some large $r$.

Theorem 4.2. Let $M$ be a complete noncompact manifold of dimension $n$, and let $p \in M$. Suppose that the Ricci curvature of $M$ satisfies $\operatorname{Ric}(x) \geq-\frac{(n-1) K}{(1+r(x))^{2}}$ for some nonnegative constant $K$, where $r(x)$ is the distance of $x$ from $p$. If $M$ has finitely many ends, so that each large end satisfies condition (VC), then the number of nonparabolic ends is equal to the number of large ends. In addition, if $M$ has a large end, then the number of large ends is also equal to the dimension of the space of bounded harmonic functions with finite Dirichlet integral on $M$.

Proof. Under the assumptions of the theorem, if we apply Theorem 1.9 to each end, then it is easy to see that the number of nonparabolic ends is equal to the number of large ends. Let $\mathscr{H}_{D}^{\infty}(M)$ be the space of bounded harmonic functions with finite Dirichlet integral, and $l \geq 1$ be the number of large ends. Recall that Theorem 1.9 and Theorem 2.1 in [24] imply that

$$
l \leq \operatorname{dim} \mathscr{H}_{D}^{\infty}(M) .
$$

More precisely, if $E_{1}, \cdots, E_{l}$ are the large ends of $M$, and $p \in M$ is a fixed point, then for each $1 \leq i \leq l$, there is a bounded harmonic function $f_{i}$ with finite Dirichlet integral so that

(1) $0<f_{i}<1$;

(2) $\lim _{r \rightarrow \infty} S_{i}(r, i)=1$, where $S_{i}(r, i)=\sup _{E_{i} \backslash B_{p}(r)} f_{i}$; and

(3) $\lim _{r \rightarrow \infty} I_{i}(r, j)=0$, for $j \neq i$, where $I_{i}(r, j)=\inf _{E_{i} \backslash B_{p}(r)} f_{j}$. 
On the other hand, Lemma 2.2 implies that if $f$ is a bounded harmonic function with finite Dirichlet integral, then $f$ is asymptotically constant at infinity on each large end. Hence there are constants $a_{1}, \ldots, a_{l}$ such that $g=f-\sum_{i=1}^{l} a_{i} f_{i}$ is asymptotically 0 at infinity of each large end. If $\sup _{M} g>0$, then the supremum must be attained at some small end. But that would imply that the small end is nonparabolic, which is impossible. Hence $\sup _{M} g=0$. Similarly, we can conclude that $\inf _{M} g=0$ and therefore $g=0$. This shows that

$$
\operatorname{dim} \mathscr{H}_{D}^{\infty}(M) \leq l
$$

and the theorem follows.

Remark 4.3. It was proved in [24] that if the Ricci curvature of $M^{n}$ at $x$ is bounded from below by $-\lambda(r(x))$, where $\lambda$ is a continuous, nonnegative and nonincreasing function on $[0, \infty)$ so that $\int_{0}^{\infty} t^{n-1} \lambda(t) d t<\infty$, then $M$ has finitely many ends.

It is easy to see that if $M$ is a complete manifold, and $f$ is a bounded harmonic function which is asymptotically constant at infinity, then $f$ must have finite Dirichlet integral near infinity. Under some additional assumptions on $M$, one may show that every bounded harmonic function must have finite Dirichlet integral.

Lemma 4.4. Let $M^{n}$ be a complete noncompact manifold with nonnegative Ricci curvature outside a compact set, and let $E$ be an end $M$. Suppose that $E$ satisfies condition (VC) and that there exists a point $p \in M$ such that $V_{p, E}(r) \geq C r^{\alpha}$ for some $C>0, \alpha>2+\frac{n-2}{n-1}$, and all $r \geq 1$. Then every bounded smooth function which is harmonic on $E$ will be asymptotically constant at infinity of $E$. In particular, such a function will have finite Dirichlet integral.

Proof. Without loss of generality, we may assume that $M$ is obtained by gluing smoothly of two copies of $E$ together. Since $E$ satisfies (VC), $M$ also does so. It is easy to see that if $E$ and hence $M$ satisfy the volume growth and the curvature assumption in the lemma, then the dimension of $M$ must be at least 3 and that $\int_{1}^{\infty} \frac{t}{V_{p}(t)} d t<\infty$. Hence by Theorem 1.9 and Theorem 2.4, $M$ admits a positive Green's function. Let $G(x, y)$ be the minimal positive Green's function. Then

$$
G(p, x) \leq C_{1} \int_{r}^{\infty} \frac{t}{V_{p}(t)} d t
$$

for some constant $C_{1}$ and all $x \in M_{r}$, where $M_{r}$ is the union of the unbounded components of $M \backslash \overline{B_{p}(r)}$. Let $f$ be a bounded function so that $\Delta f=0$ on $M \backslash B_{p}(R)$ for some $R>1$. The gradient estimate 
(Lemma 1.5) implies that $|\nabla f|(x) \rightarrow 0$ as $r(x) \rightarrow \infty$, where $r(x)$ is the distance of $x$ from $p$. On the other hand, since $M$ has nonnegative Ricci curvature outside a compact set, which may be assumed to be $B_{p}(R)$, the Bochner formula [Y] asserts that $|\nabla f|^{(n-2) /(n-1)}$ is subharmonic on $M \backslash \overline{B_{p}(R)}$ in the sense of distribution. Therefore the maximum principle yields that

$$
|\nabla f|^{(n-2) /(n-1)}(x) \leq C_{2} G(p, x)
$$

on $M \backslash \overline{B_{p}(r)}$ for $r>R$ and some constant $C_{2}>0$. By the assumption on the volume growth of $M,(4.2)$ and (4.3), we conclude that

$$
|\nabla f|(x) \leq C_{3} r^{(2-\alpha) \cdot(n-1) /(n-2)} \leq C_{3} r^{-\beta}
$$

for some constants $C_{3}>0$ and $\beta>1$, and for all $x$ in an unbounded component of $M \backslash \overline{B_{p}(r)}$. Hence for $r>R$, we have

$$
\begin{aligned}
\int_{B_{p}(r) \backslash B_{p}(R)}|\nabla f|^{2} & \leq \int_{\left(M \backslash M_{r}\right) \backslash B_{p}(R)}|\nabla f|^{2} \\
& =-\int_{\partial B_{p}(R)} f \frac{\partial f}{\partial r}+\int_{\partial M_{r}} f \frac{\partial f}{\partial r} \\
& \leq C_{4}\left(1+r^{-\beta} A_{p}(r)\right),
\end{aligned}
$$

for some constant $C_{4}>0$, where $A_{p}(r)$ is the area of $\partial B_{p}(r)$. By the assumption that $M$ has nonnegative Ricci curvature outside a compact set, it is not hard to prove that $r A(r) \leq C_{5} V_{p}(r)$ for some constant $C_{5}>0$. Using Lemma $1.6,(4.5)$ and the fact that $|\nabla f|^{2}$ is subharmonic outside $B_{p}(R)$, we obtain

$$
|\nabla f|^{2}(x) \leq \frac{C_{6}}{V_{p}(r)}\left(1+r^{-\beta-1} V_{p}(r)\right)
$$

for some constant $C_{6}>0$, all $r>2 R$ and $x \in \partial B_{p}(r)$, where we have used Lemma 1.3 and the fact that $M$ satisfies condition (VC). Since $V_{p}(r) \geq C r^{\alpha}$,

$$
|\nabla f|^{2}(x) \leq C_{7}\left(r^{-\alpha}+r^{-\beta-1}\right)
$$

for some constant $C_{7}>0$. Since $\alpha>2$ and $\beta>1$, using Lemma 2.1, Lemma 1.4, and arguing as in the proof of Lemma 2.2, we conclude that $f$ is asymptotically constant.

Theorem 4.5. Let $M$ be a complete noncompact manifold with nonnegative Ricci curvature outside a compact set. Let us assume that $M$ has 
a large end. Suppose that each large end of $M$ satisfies condition (VC) and the volume growth condition in Lemma 4.4. Then the number of large ends is equal to the dimension of the space of bounded harmonic functions. In particular, every bounded harmonic function will have finite Dirichlet integral

Proof. Use Lemma 4.4, and proceed as in the proof of Theorem 4.2.

We will take this opportunity to point out that if $M$ has small volume growth, then any bounded function which is harmonic outside a compact set must have finite Dirichlet integral without any curvature assumption.

Proposition 4.6. Let $M$ be a complete noncompact manifold, such that $\int_{1}^{\infty} \frac{t}{V_{p}(t)} d t=\infty$, for some point $p \in M$. If $f$ is a bounded smooth function so that $f$ is harmonic outside $B_{p}(R)$ for some $R>0$, then $f$ has finite Dirichlet integral.

Proof. Without loss of generality, we may assume that $f>0$. Since $f$ is harmonic outside $B_{p}(R)$, and $\int_{1}^{\infty} \frac{t}{V_{p}(t)} d t=\infty$, we claim that for all $r_{1}>R$ and for $r_{2}>r_{1}$ sufficiently large, we have

$$
\sup _{\partial B_{p}\left(r_{2}\right)} f \geq \inf _{\partial B_{p}\left(r_{1}\right)} f \text {. }
$$

If this is not true, then we can find a harmonic function on $M \backslash B_{p}(R)$ bounded between 0 and 1 , which is equal to 1 on $\partial B_{p}(R)$ and has infimum equal to 0 . However, this will imply that $M$ admits a positive Green's function, which is impossible by Theorem 1.9. For $r_{1}>R$, observe that $f^{2}$ is subharmonic outside $B_{p}(R)$. From (4.6) and Corollary 2.4 of [22] it follows that

$$
\left(\int_{\partial B_{p}\left(r_{1}\right)} \frac{\partial\left(f^{2}\right)}{\partial r}\right)\left(\int_{r_{1}}^{r_{2}} \frac{t}{V_{p}(t)} d t\right) \leq C_{8}\left(\sup _{\partial B_{p}\left(r_{2}\right)} f^{2}-\inf _{\partial B_{p}\left(r_{1}\right)} f^{2}\right),
$$

for some constant $C_{8}>0$ independent of $r_{2}$. Letting $r_{2} \rightarrow \infty$ and using the boundedness of $f$ and $\int_{1}^{\infty} \frac{t}{V_{p}(t)} d t=\infty$, we have

$$
\int_{\partial B_{p}\left(r_{1}\right)} \frac{\partial\left(f^{2}\right)}{\partial r} \leq 0
$$

Hence

$$
\int_{B_{p}\left(r_{1}\right)} \Delta\left(f^{2}\right) \leq 0
$$

and

$$
\int_{B_{p}(R)} \Delta\left(f^{2}\right)+2 \int_{B_{p}\left(r_{1}\right) \backslash B_{p}(R)}|\nabla f|^{2} \leq 0,
$$


where we have used the fact that $f$ is harmonic outside $B_{p}(R)$. Hence the proposition follows by letting $r_{1} \rightarrow \infty$.

\section{Volume comparison}

In this section, we will discuss the validity of condition (VC). As mentioned in $\S 3$, if $M$ has nonnegative Ricci curvature everywhere, then $M$ satisfies condition (VC). If $M$ has asymptotically nonnegative sectional curvature, then each end of $M$ satisfies condition (VC). If we only assume that $M$ has nonnegative Ricci curvature outside a compact set, then we know $M$ has only finitely many ends; see [24], for example. In that case, it is still an open question as of whether an end of $M$ will satisfy condition (VC), even if we assume that $M$ has only one end. However, using the method in [26], we can prove the following weaker version of the volume comparison.

Proposition 5.1. Let $M^{n}$ be a complete Riemannian manifold with nonnegative Ricci curvature outside $B_{p}(1)$. Then there exists a constant $C_{1}>1$ depending only on $M$ such that for all $R>0$, there exists a point $x \in \partial B_{p}(R)$ satisfying

$$
V_{p}(R) \leq C_{1} V_{x}(R / 5)
$$

Proof. It is sufficient to prove the theorem for $R$ large. Following the argument in [26], let us divide $\partial B_{p}(2)$ into $m$ subsets $\left\{U_{1}, \cdots, U_{m}\right\}$ such that $\operatorname{diam}\left(U_{i}\right) \leq 2$. Let $K_{i}$ be the set of points $x$ outside $B_{p}(2)$ such that $x$ lies on some minimal geodesic emanating from $p$ that intersects $U_{i}$. For $x$ and $y$ in $K_{i}$, the triangle inequality implies that any minimal geodesic from $x$ to $y$ will not meet $B_{p}(1)$. Let us assume that $K_{1}, \cdots, K_{l}$ are unbounded and that $K_{l+1}, \cdots, K_{m}$ are bounded. There exists $R_{0}>2$ such that for any $R>R_{0}$, there is $l+1 \leq i_{R} \leq m$ such that the volume of $\left(K_{i_{R}} \cap B_{p}(R)\right)$ is larger than $\frac{1}{2 m} V_{p}(R)$. Since $K_{i_{R}}$ is unbounded, there exists $x \in K_{i_{R}} \cap \partial B_{p}(R)$. Moreover, because every minimal geodesic from $x$ to any point in $K_{i_{R}} \cap B_{p}(R)$ does not meet $B_{p}(1)$, and $M$ has nonnegative Ricci curvature outside $B_{p}(1)$, the Bishop comparison theorem (see [8]) implies that

$$
V_{x}\left(\frac{R}{5}\right) \geq C_{2} \operatorname{Vol}\left(K_{i_{R}} \cap B_{p}(R)\right) \geq \frac{C_{2}}{2 m} V_{p}(R),
$$

where $C_{2}>0$ is a constant depending only on $n$. This completes the proof of the proposition.

The following theorem was proved by Cai, Colding, and Yang [5]. 
Theorem (Cai-Colding-Yang). For any integer $n \geq 2$, there exists $\epsilon(n)$ $>0$ depending only on $n$ such that if $M$ is a complete noncompact manifold of dimension $n$ with Ricci curvature bounded from below by $-(n-1) \Lambda^{2}$ for some $\Lambda \geq 0$, and with nonnegative Ricci curvature outside $B_{p}(a)$ for some point $p \in M$. If $\Lambda a \leq \epsilon(n)$, then $M$ has at most two ends.

In the rest of this section, we would like to study the volume comparison property on this category of manifolds. First, let us recall a lemma in [5] and a lemma in [4].

Lemma 5.2. Let $M^{n}$ be a complete noncompact Riemannian manifold with Ricci curvature bounded from below by $-(n-1)$. Then there exists two constants $\epsilon=\epsilon(n)>0$ and $1>\delta=\delta(n)>0$ depending only on $n$, such that if $u$ is a function on $M$ with the following properties:

(1) $u(p)=0$;

(2) $u \geq-2 \epsilon$;

(3) $|\nabla u| \leq 2$; and

(4) $\Delta u \leq 2(n-1)$ in the barrier sense,

then $u(x)<2(1-\delta)-4 \epsilon$ for all $x \in \partial B_{p}((1-\delta))$.

Proof. This is Lemma 2.3 in [5].

Lemma 5.3. Let $M^{n}$ be a complete manifold with nonnegative Ricci curvature outside $B_{p}(a)$ for some point $p$ and $a>0$. Then $M$ cannot admit a line $\gamma$ parametized by arclength so that

$$
d\left(\gamma(t), B_{p}(a)\right) \geq|t|+a .
$$

Proof. This is Lemma 3.3 in [4].

Using the method of proof as in Proposition 2.2 of [4] and the theorem in [5], one can prove the following lemma.

Lemma 5.4. Let $M^{n}$ be a complete manifold with Ricci curvature bounded from below by $-(n-1)$ and has nonnegative Ricci curvature on $M \backslash B_{p}\left(\frac{a}{2}\right)$ for some point $p$. Suppose that $a<\frac{1}{3} \epsilon$, where $\epsilon(n)$ is the constant in Lemma 5.2. Let $0<\eta \leq \frac{1}{5}$ be a fixed constant and let $K$ be any compact set. Then there exists $t_{0}>0$ such that for all $t \geq t_{0}$ and any three points $x, y$, and $z$ in $B_{p}((1+\eta) t) \backslash B_{p}((1-\eta) t)$, if $\alpha_{x y}, \alpha_{y z}$, and $\alpha_{z x}$ are minimal geodesics from $x$ to $y, y$ to $z$, and $z$ to $x$, respectively, then at least one of the minimal geodesics will NOT intersect $K$.

Proof. We may assume that $K=B_{p}(R)$ for some $R>0$, and will prove the lemma by contradiction. Suppose that there exist $t_{k} \rightarrow \infty, x_{k}$, $y_{k}, z_{k}$ in $B_{p}\left((1+\eta) t_{k}\right) \backslash B_{p}\left((1-\eta) t_{k}\right)$, and minimal geodesics $\alpha_{x_{k} y_{k}}$, $\alpha_{y_{k} z_{k}}$, and $\alpha_{z_{k} x_{k}}$ from $x_{k}$ to $y_{k}, y_{k}$ to $z_{k}$, and $z_{k}$ to $x_{k}$ respectively, such that each of them intersects $B_{p}(R)$. Let $\gamma_{1, k}, \gamma_{2, k}$, and $\gamma_{3, k}$ be 
minimal geodesics from $p$ to $x_{k}, y_{k}$, and $z_{k}$ respectively. Passing to subsequences, we may assume that $\gamma_{1, k} \rightarrow \gamma_{1}, \gamma_{2, k} \rightarrow \gamma_{2}$, and $\gamma_{3, k} \rightarrow \gamma_{3}$ for some rays $\gamma_{i}, \quad 1 \leq i \leq 3$, emanating from $p$. The convergence is uniform on compact subsets of $M$. We claim that

$$
d\left(\gamma_{i}\left(s_{1}\right), \gamma_{j}\left(s_{2}\right)\right) \geq s_{1}+s_{2}-6 a,
$$

for all $s_{1}, s_{2}>0$, and $1 \leq i<j \leq 3$. Let us prove that

$$
d\left(\gamma_{1}\left(s_{1}\right), \gamma_{2}\left(s_{2}\right)\right) \geq s_{1}+s_{2}-6 a
$$

for all $s_{1}, s_{2}>0$, and the remaining cases are similar. Let us denote $\alpha_{x_{k} y_{k}}$ by $\alpha_{k}$, and let $l_{k}$ be its length. Let $r(x)$ to be the distance from a point $x$ to $p$. Suppose

$$
d\left(\gamma_{1}\left(s_{1}\right), \gamma_{2}\left(s_{2}\right)\right)<s_{1}+s_{2}-6 a,
$$

for some $s_{1}, s_{2}>0$. Then for all $k$ sufficiently large,

$$
d\left(\gamma_{1, k}\left(s_{1}\right), \gamma_{2, k}\left(s_{2}\right)\right)<s_{1}+s_{2}-6 a \text {. }
$$

Hence

$$
\begin{aligned}
l_{k} & =d\left(x_{k}, y_{k}\right) \\
& \leq d\left(x_{k}, \gamma_{1, k}\left(s_{1}\right)\right)+d\left(\gamma_{1, k}\left(s_{1}\right), \gamma_{2, k}\left(s_{2}\right)\right)+d\left(\gamma_{2, k}\left(s_{2}\right), y_{k}\right) \\
& <r\left(x_{k}\right)-s_{1}+s_{1}+s_{2}-6 a+r\left(y_{k}\right)-s_{2} \\
& =r\left(x_{k}\right)+r\left(y_{k}\right)-6 a .
\end{aligned}
$$

Let $m_{k}$ be a point on $\alpha_{k}$ such that

$$
r\left(x_{k}\right) d\left(m_{k}, y_{k}\right)=r\left(y_{k}\right) d\left(m_{k}, x_{k}\right) .
$$

Let $p_{k} \in \alpha_{k} \cap B_{p}(R)$ which is nonempty by assumption. Without loss of generality, we may assume that $d\left(p_{k}, y_{k}\right) \leq d\left(m_{k}, y_{k}\right)$. Then

$$
\begin{aligned}
d\left(p, m_{k}\right) & \leq d\left(p, p_{k}\right)+d\left(p_{k}, m_{k}\right) \\
& \leq R+d\left(m_{k}, y_{k}\right)-d\left(p_{k}, y_{k}\right) \\
& \leq R+d\left(m_{k}, y_{k}\right)-r\left(y_{k}\right)+R \\
& =\frac{r\left(y_{k}\right)}{r\left(x_{k}\right)+r\left(y_{k}\right)} l_{k}-r\left(y_{k}\right)+2 R \\
& <\frac{r\left(y_{k}\right)}{r\left(x_{k}\right)+r\left(y_{k}\right)}\left(r\left(x_{k}\right)+r\left(y_{k}\right)-6 a\right)-r\left(y_{k}\right)+2 R, \\
& =2 R-\frac{6 a \cdot r\left(y_{k}\right)}{r\left(x_{k}\right)+r\left(y_{k}\right)},
\end{aligned}
$$


where we have used the fact that $d\left(p_{k}, y_{k}\right) \leq d\left(m_{k}, y_{k}\right),(5.2)$, and (5.3). Since $x_{k}$ and $y_{k}$ are in $B_{p}\left((1+\eta) t_{k}\right) \backslash B_{p}\left((1-\eta) t_{k}\right)$ and $0<\eta \leq \frac{1}{5}$, we have

$$
\frac{r\left(y_{k}\right)}{r\left(x_{k}\right)+r\left(y_{k}\right)} \geq \frac{1-\eta}{2(1+\eta)} \geq \frac{1}{3} \text {. }
$$

Combining this with (5.4) and (5.5), we conclude

$$
d\left(p, m_{k}\right)<2 R-\frac{6 a(1-\eta)}{2(1+\eta)} \leq 2 R-2 a .
$$

In particular, $m_{k} \in B_{p}(2 R)$. We may reparametrized $\alpha_{k}$ with arclength, so that $\alpha_{k}(0)=m_{k}, \alpha\left(-d\left(m_{k}, x_{k}\right)\right)=\alpha_{k}\left(x_{k}\right)$, and $\alpha\left(d\left(m_{k}, y_{k}\right)\right)=$ $\alpha_{k}\left(y_{k}\right)$. Note that $d\left(m_{k}, x_{k}\right) \rightarrow \infty$ and $d\left(m_{k}, y_{k}\right) \rightarrow \infty$. Without loss of generality, we may assume that $\alpha_{k}$ converges to a line $\gamma$ in $M$. For any $s>0$, and for $k$ sufficiently large

$$
\begin{aligned}
d\left(p, \alpha_{k}(s)\right) & \geq d\left(p, y_{k}\right)-d\left(y_{k}, \alpha_{k}(s)\right) \\
& =r\left(y_{k}\right)-d\left(m_{k}, y_{k}\right)+s \\
& =r\left(y_{k}\right)-\frac{r\left(y_{k}\right)}{r\left(x_{k}\right)+r\left(y_{k}\right)} l_{k}+s \\
& \geq r\left(y_{k}\right)-\frac{r\left(y_{k}\right)}{r\left(x_{k}\right)+r\left(y_{k}\right)}\left(r\left(x_{k}\right)+r\left(y_{k}\right)-6 a\right)+s \\
& =s+\frac{6 a \cdot r\left(y_{k}\right)}{r\left(x_{k}\right)+r\left(y_{k}\right)} \\
& \geq s+2 a,
\end{aligned}
$$

where we have used (5.2) and (5.5). Hence by letting $k \rightarrow \infty$, we have

$$
d\left(\gamma(s), B_{p}(a / 2)\right) \geq|s|+a
$$

for $s>0$. Similarly, one can prove that (5.6) is true for $s<0$. Since $\gamma$ is a line, and $M$ has nonnegative Ricci curvature outside $B_{p}\left(\frac{a}{2}\right),(5.6)$ contradicts Lemma 5.3. Hence (5.1) is true.

Let us now consider the Busemann functions

$$
b_{i}(x)=\lim _{t \rightarrow \infty}\left(d\left(\gamma_{i}(t), x\right)-t\right),
$$

for $i=1,2$ and $x \in M$. Let $u=b_{1}+b_{2}$. Then

(1) $u(p)=0$

(2) $u \geq-2 \epsilon$;

(3) $|\nabla u| \leq 2$;

(4) $\Delta u \leq 2(n-1)$, in the barrier sense. 
Properties (1) and (3) are obvious. Property (4) is derived from the fact that the Ricci curvature is bounded from below by $-(n-1)$ (see [12]), and property (2) is derived from (5.1) and the assumption that $a<\frac{\epsilon}{3}$. Lemma 5.2 now implies that

$$
u(x)<2(1-\delta)-4 \epsilon
$$

for all $x \in \partial B_{p}((1-\delta))$, where $\delta$ is the constant in Lemma 5.2 that depends only on $n$. In particular,

$$
u\left(\gamma_{3}(1-\delta)\right)<2(1-\delta)-4 \epsilon
$$

However, by (5.1) again,

$$
\begin{aligned}
& d\left(\gamma_{1}(t), \gamma_{3}(1-\delta)\right)+d\left(\gamma_{2}(t), \gamma_{3}(1-\delta)\right)-2 t \\
& \geq 2 t+2(1-\delta)-12 a-2 t \geq 2(1-\delta)-4 \epsilon,
\end{aligned}
$$

where we have used the assumption that $a<\frac{1}{3} \epsilon$. So

$$
u\left(\gamma_{3}(1-\delta)\right) \geq 2(1-\delta)-4 \epsilon,
$$

which contradicts (5.7). Hence the lemma is true.

Proposition 5.5. Let $M^{n}$ be a complete manifold with Ricci curvature bounded from below by $-(n-1)$ and has nonnegative Ricci curvature on $M \backslash B_{p}(a)$ for some point $p$. Suppose that $a<\frac{1}{12} \epsilon(n)$, where $\epsilon(n)$ is the constant in Lemma 5.2. If $M$ has two ends, then each end of $M$ satisfies condition (VC).

Proof. Let $R_{0}>0$ so that $M \backslash B_{p}\left(R_{0}\right)$ has exactly two unbounded components $E_{1}$ and $E_{2}$. We would like to prove that there exists $R_{1}$, such that for all $x, y \in E_{i} \backslash B_{p}\left(R_{1}\right)$, any minimal geodesic from $x$ to $y$ will not intersect $B_{p}(a)$ for each $i=1,2$. Let us assume the contrary. Then there exist $x_{k}, y_{k} \in E_{1}$, and a minimal geodesic $\alpha_{k}$ from $x_{k}$ to $y_{k}$ so that $\lim _{k \rightarrow \infty} d\left(p, x_{k}\right)=\infty, \lim _{k \rightarrow \infty} d\left(p, y_{k}\right)=\infty$, and $\alpha_{k} \cap B_{p}(a) \neq \varnothing$. We may assume that $\alpha_{k}$ converges to a line $\gamma$ so that $\gamma \cap \overline{B_{p}(a)} \neq \varnothing$. Let $q$ be a point in $\gamma \cap \overline{B_{p}(a)}$. We may assume that $\gamma(0)=q$ and $\gamma$ is parametrized by arclength. Let $\gamma_{1}(t)=\gamma(t)$ for $t \geq 0$ and $\gamma_{2}(t)=\gamma(-t)$ for $t \leq 0$. Then $\gamma_{1}$ and $\gamma_{2}$ are rays from $q$. Let $\gamma_{3}$ be a ray from $q$ which will be in $E_{2}$ for sufficiently large $t$. By construction, it is easy to see that if $t$ is large, then there are minimal geodesics from $\gamma_{i}(t)$ to $\gamma_{j}(t)$ for any $1 \leq i<j \leq 3$, so that they all intersect $B_{p}\left(R_{0}\right)$. Since $B_{p}(a) \subset$ $B_{q}(2 a)$ and $a<\frac{1}{12} \epsilon$, this contradicts Lemma 5.4. The proposition then follows from the volume comparison theory in [8], the fact that $M$ has nonnegative Ricci curvature outside $B_{p}(a)$ and Proposition 5.1. 
In case $M$ has only one end, we have a weaker result. Let us first prove a simple lemma.

Lemma 5.6. Let $M^{n}$ be a complete manifold with Ricci curvature bounded from below by $-(n-1)$ and has nonnegative Ricci curvature outside $B_{p}(a)$ for some point $p$. Suppose $a<\frac{1}{6} \epsilon(n)$, where $\epsilon(n)$ is the constant in Lemma 5.2. Then there exists a constant $C_{3}>1$ depending only on $n$, and there exists $t_{0}$ such that for all $t \geq t_{0}$ and $x, y, z \in \partial B_{p}(t)$, we have either

(1) $C_{3}^{-1} \leq V_{x}\left(\frac{t}{5}\right) / V_{y}\left(\frac{t}{5}\right) \leq C_{3}$;

(2) $C_{3}^{-1} \leq V_{y}\left(\frac{t}{5}\right) / V_{z}\left(\frac{t}{5}\right) \leq C_{3}$; or

(3) $C_{3}^{-1} \leq V_{z}\left(\frac{t}{5}\right) / V_{x}\left(\frac{t}{5}\right) \leq C_{3}$.

Proof. Let $t_{0}$ be the number in Lemma 5.4 with $\eta=\frac{1}{5}$, and $K=$ $\overline{B_{p}(a)}$. Let $t \geq t_{0}$, and $x, y, z \in \partial B_{p}(t)$. Without loss of generality, we may assume that $V_{z}\left(\frac{t}{5}\right) \geq V_{y}\left(\frac{t}{5}\right) \geq V_{x}\left(\frac{t}{5}\right)$. Suppose for all $y^{\prime} \in B_{y}\left(\frac{t}{5}\right)$, there exists a minimal geodesic from $x$ to $y^{\prime}$, which does not intersect $B_{p}(a)$. Then by the Bishop comparison theorem, we have

$$
V_{y}\left(\frac{t}{5}\right) \leq \frac{\left(2 t+\frac{t}{5}\right)^{n}}{\left(\frac{t}{5}\right)^{n}} V_{x}\left(\frac{t}{5}\right)=11^{n} V_{x}\left(\frac{t}{5}\right)
$$

and hence

$$
1 \leq \frac{V_{y}\left(\frac{t}{5}\right)}{V_{x}\left(\frac{t}{5}\right)} \leq 11^{n}
$$

Suppose there exist $y_{1} \in B_{y}\left(\frac{t}{5}\right)$ and a minimal geodesic from $x$ to $y_{1}$ so that it intersects $B_{p}(a)$. Then by Lemma 5.4, for all $z^{\prime} \in B_{z}\left(\frac{t}{5}\right)$, there is a minimal geodesic from $x$ to $z^{\prime}$ or a minimal geodesic from $y_{1}$ to $z^{\prime}$, which does not intersect $B_{p}(a)$. Again the Bishop comparison theorem implies that

$$
\begin{aligned}
V_{z}\left(\frac{t}{5}\right) & \leq 11^{n}\left(V_{x}\left(\frac{t}{5}\right)+V_{y_{1}}\left(\frac{t}{5}\right)\right) \\
& \leq 11^{n} 2^{n}\left(V_{x}\left(\frac{t}{5}\right)+V_{y}\left(\frac{t}{5}\right)\right) \\
& \leq 2 \cdot 22^{n} V_{y}\left(\frac{t}{5}\right),
\end{aligned}
$$

where we have used the facts that $V_{x}\left(\frac{t}{5}\right) \leq V_{y}\left(\frac{t}{5}\right)$ and $y_{1} \in B_{y}\left(\frac{t}{5}\right)$. Since $V_{z}\left(\frac{t}{5}\right) \geq V_{y}\left(\frac{t}{5}\right)$, combining it with (5.8) shows that the lemma is valid for $C_{3}=2 \cdot 22^{n}$. 
Proposition 5.7. Let $M^{n}$ be a complete manifold with Ricci curvature bounded from below by $-(n-1)$ and has nonnegative Ricci curvature on $M \backslash B_{p}(a)$ for some point $p$. Suppose $a<\frac{1}{6} \epsilon(n)$, where $\epsilon(n)$ is the constant in Lemma 5.2. If $M$ has only one end, then there exist a constant $C_{4}>0$ and a sequence $r_{k} \rightarrow \infty$ such that for all $x \in \partial B_{p}\left(r_{k}\right)$, we have

$$
V_{p}\left(r_{k}\right) \leq C_{4} V_{x}\left(\frac{r_{k}}{5}\right)
$$

Proof. Let $C_{3}$ and $t_{0}$ be the constants in Lemma 5.6, and let $C_{1}$ be the constant in Proposition 5.1 for $M$. Suppose for all $t$ sufficiently large and for $x \in \partial B_{p}(t)$ we have

$$
\frac{1}{2} C_{1}^{-1} C_{3}^{-1} V_{p}(t) \leq V_{x}\left(\frac{t}{5}\right) .
$$

Then we are done. Otherwise, there is a sequence $t_{k} \rightarrow \infty$ and $x_{k} \in$ $\partial B_{p}\left(t_{k}\right)$ such that

$$
V_{x_{k}}\left(\frac{t_{k}}{5}\right)<\frac{1}{2} C_{1}^{-1} C_{3}^{-1} V_{p}\left(t_{k}\right)
$$

for all $k$. By Proposition 5.1, there exists $y_{k} \in \partial B_{p}\left(t_{k}\right)$ such that

$$
V_{p}\left(t_{k}\right) \leq C_{1} V_{y_{k}}\left(\frac{t_{k}}{5}\right)
$$

for all $k$. Since $M$ has only one end, passing to a subsequence if necessary, we may assume that $x_{k}$ and $y_{k}$ are both in the unbounded component of $M \backslash \bar{B}_{p}\left(R_{k}\right)$ for some $R_{k} \leq t_{k}$ and $R_{k} \rightarrow \infty$. For each $k$ there is a continuous curve $\sigma_{k}(s)$, for $0 \leq s \leq l$, such that $\sigma_{k}(0)=x_{k}$, $\sigma_{k}(l)=y_{k}$, and $\sigma_{k}(s) \in M \backslash \bar{B}_{p}\left(R_{k}\right)$ for all $s$. Let $r(s)=d\left(p, \sigma_{k}(s)\right)$ and let $V_{s}$ be the volume of $B_{\sigma_{k}(s)}\left(\frac{r(s)}{5}\right)$. It is easy to see that $V_{s}$ is a continuous function of $s$. Let us define

$$
A=\left\{s \mid 0 \leq s \leq l, V_{s}<\frac{1}{2} C_{1}^{-1} C_{2}^{-1} V_{p}(r(s))\right\},
$$

which is an open set in the interval $[0, l]$ since $V_{s}$ and $V_{p}(r(s))$ are continuous in $s$. Inequality (5.10) asserts that $0 \in A$. Let $s^{*}$ be the supremum of $s$ such that $[0, s) \subset A$. Then $0<s^{*}<l$ by $(5.11)$ and the fact that $C_{3}>1$. Clearly, from the definition of $A$, we have

$$
V_{s^{*}}=\frac{1}{2} C_{1}^{-1} C_{3}^{-1} V_{p}\left(r\left(s^{*}\right)\right)
$$


Let us set $r_{k}=r\left(s^{*}\right)$. Proposition 5.1 implies that there is a point $y \in$ $\partial B_{p}\left(r_{k}\right)$ such that

$$
V_{y}\left(\frac{r_{k}}{5}\right) \geq C_{1}^{-1} V_{p}\left(r_{k}\right) .
$$

By (5.12) and (5.13), we have

$$
V_{y}\left(\frac{r_{k}}{5}\right) \geq 2 C_{3} V_{s^{*}}
$$

Hence from Lemma 5.6 and (5.11) it follows that, for all $x \in \partial B_{p}\left(r_{k}\right)$,

$$
V_{x}\left(\frac{r_{k}}{5}\right) \geq C_{3}^{-1} V_{s^{*}} \geq \frac{1}{2} C_{3}^{-2} C_{1}^{-1} V_{p}\left(r_{k}\right),
$$

which completes the proof of the proposition.

We would like to mention that it is still unclear that whether Proposition 5.7 is true for all $r$ rather than for a sequence of $r_{k} \rightarrow \infty$. It is also not known that whether a complete noncompact manifold with nonnegative Ricci curvature outside a compact set with only one end will satisfy condition (VC).

\section{Nonnegatively curved manifolds with finite first Betti number}

In this section, we will consider complete noncompact manifolds with nonnegative Ricci curvature outside a compact set which has finite first Betti number. We will show that for this kind of manifolds, condition (VC) is satisfied by each end, and that Theorem 1.9 is valid and the results in [20] can be carried over to this case. Let us first begin with a lemma.

Lemma 6.1. Let $M$ be a complete manifold with $k$ ends. Suppose that the first Betti number $b_{1}(M)$ of $M$ is finite. For $R>0$ sufficiently large, and for $r>0$, let $A_{p}(R, R+r)$ denote the annulus $B_{p}(R+r) \backslash \overline{B_{p}(R)}$, and let $M_{R}$ be the union of all the unbounded components of $M \backslash \overline{B_{p}(R)}$. Then $A_{p}(R, R+r) \cap M_{R}$ has exactly $k$ connected components.

Proof. Let $\left\{\left[\gamma_{1}\right], \cdots,\left[\gamma_{b}\right]\right\}$ be a set of basis for $H_{1}(M)$, where $b=$ $b_{1}(M)$. By finiteness, there exists $R_{0}$ sufficiently large such that $\gamma_{i} \subset$ $B_{p}\left(R_{0}\right)$ for all $1 \leq i \leq b$. In particular, any element $[\gamma] \in H_{1}(M)$ can be represented in the form $\sum_{i}^{b} a_{i} \gamma_{i} \subset B_{p}\left(R_{0}\right)$. Since $k$ is the number of ends of $M$, there exists $R_{1}$ such that for $R>R_{1}, M \backslash \overline{B_{p}(R)}$ has exactly $k$ unbounded components. We claim that if we choose $R>\max \left\{R_{0}, R_{1}\right\}$, then $A_{p}(R, R+r) \cap M_{R}$ has exactly $k$ components. Let $U$ be the union of $B_{p}(R+r)$ and the bounded components of $M \backslash \overline{B_{p}(R)}$. Then $U$ is 
connected and $M_{R}$ has exactly $k$ components. Moreover, $M=M_{R} \cup U$. Let us consider the Mayer-Vietoris sequence

$$
\begin{gathered}
H_{1}(U) \oplus H_{1}\left(M_{R}\right) \stackrel{j_{*}}{\rightarrow} H_{1}(M) \stackrel{\partial_{*}}{\rightarrow} H_{0}\left(U \cap M_{R}\right) \\
\stackrel{i_{*}}{\rightarrow} H_{0}(U) \oplus H_{0}\left(M_{R}\right) \stackrel{j_{*}^{\prime}}{\rightarrow} H_{0}(M) .
\end{gathered}
$$

Since all the reprsentatives of $H_{1}(M)$ lie inside $U$, the map $j_{*}$ is surjective. The map $j_{*}^{\prime}$ is obviously also surjective. Since $U$ is connected and $M_{R}$ has exactly $k$ components, we have the exact sequence

$$
0 \rightarrow H_{0}\left(U \cap M_{R}\right) \rightarrow \overbrace{\mathbb{Z} \oplus \cdots \oplus \mathbb{Z}}^{k+1} \rightarrow \mathbb{Z} \rightarrow 0 .
$$

Hence $H_{0}\left(U \cap M_{R}\right)$ is isomorphic to the kernel of the surjective map $\underbrace{\mathbb{Z} \oplus \cdots \oplus \mathbb{Z}}_{k+1} \rightarrow \mathbb{Z}$, which is easily seen to be $\underbrace{\mathbb{Z} \oplus \cdots \oplus \mathbb{Z}}_{k}$. Hence $A_{p}(R, R+r)=U \cap M_{R}$ has exactly $k$ components.

Corollary 6.2. Let $M$ be a complete noncompact manifold with nonnegative Ricci curvature outside a compact set and let $p$ be a fixed point. Suppose $b_{1}(M)$ is finite. Given an end $E$, there exists a constant $C>0$ such that for any $R$ sufficiently large, we have

$$
V_{p, E}(R) \leq C V_{x}(R / 5)
$$

for all $x \in \partial B_{p}(R) \cap \overline{E_{R}}$, where $V_{p, E}(R)$ is the volume of $B_{p}(R) \cap E$, and $E_{R}$ is the unbounded component of $E \cap\left(M \backslash \overline{B_{p}(R)}\right)$.

Proof. Let us first observe that Theorem 3.1 of [24] asserts that $M$ has finitely many ends. Hence the statement of the corollary is meaningful. Since the statement of the corollary is localized at an end $E$, we may assume that $M$ is obtained by gluing two copies of $E$ together. Let us also assume that $M \backslash B_{p}(a)$ has nonnegative Ricci curvature for some point $p \in M$ and some $a>0$. By the proof of Proposition 5.1, it is easy to see that there is a constant $C$ such that for all $R>0$ there exists a point $x \in \partial B_{p}(R) \cap M_{R / 2}$, so that

$$
V_{p}(R) \leq C V_{x}(R / 5)
$$

where $M_{R / 2}$ is the union of the unbounded components of $M \backslash \overline{B_{p}\left(\frac{R}{2}\right)}$. Since the ends are symmetric, the existence of such a point occurs at both ends. For the sake of convenience, let us just denote a fixed end by $E$. To prove the corollary, it suffices to show that there exists a constant $C>0$ such that for all $y \in B_{p}(R) \cap M_{R / 2}$ we have

$$
V_{x}(R / 5) \leq C V_{y}(R / 5) \text {. }
$$


The ball covering theorem of [26] asserts that the set $B_{p}(R) \cap M_{R / 2}$ and hence $\partial B_{p}(R) \cap \overline{E_{R}}$ can be covered by $N$ number of balls $B_{y_{i}}\left(\frac{R}{5}\right)$ with centers $y_{i} \in B_{p}(R) \cap M_{\frac{R}{2}}$, where $N$ depends only on the lower bound of the Ricci curvature of $B_{p}(a)$ and $a$. The fact that $\left\{B_{y_{i}}\left(\frac{R}{5}\right)\right\}$ covers $\partial B_{p}(R) \cap \overline{E_{R}}$ implies that $y_{i} \notin B_{p}\left(\frac{4 R}{5}\right)$.

Let us first consider two balls $B_{y_{i}}\left(\frac{R}{5}\right)$ and $B_{y_{j}}\left(\frac{R}{5}\right)$ such that $\overline{B_{y_{i}}\left(\frac{R}{5}\right)} \cap$ $\overline{B_{y_{j}}\left(\frac{R}{5}\right)} \neq \varnothing$. Clearly, any geodesic joining $y_{i}$ to a point in $B_{y_{j}}\left(\frac{R}{5}\right)$ must have length at most $\frac{3 R}{5}$, and therefore will not intersect $B_{p}(a)$ for $R>$ 5a. Thus the Bishop comparison theorem implies that

$$
V_{y_{j}}(R / 5) \leq C V_{y_{i}}(R / 5) \text {. }
$$

By Lemma 6.1, the set $\partial B_{p}(R) \cap \overline{E_{R}}$ is connected. Therefore $\bigcup_{i} B_{y_{i}}\left(\frac{R}{5}\right)$ is connected. Since the volume of any two adjacent balls can be compared, this shows that

$$
V_{y_{j}}(R / 5) \leq C^{N} V_{y_{k}}(R / 5)
$$

for all $y_{j}$ and $y_{k}$, and hence the corollary is proved.

We are now ready to prove some estimates on the Green's function which are similar to those in Theorem 1.9. Since the condition (VC) is valid only for those $x \in E \cap\left(M \backslash \overline{B_{p}(R)}\right)$, the estimate is only valid for those points also.

Theorem 6.3. Let $M$ be a complete noncompact manifold with nonnegative Ricci curvature outside a compact set. Suppose that the first Betti number $b_{1}(M)$ of $M$ is finite. Then the following hold:

(1) Let $E$ be an end of $M$ and let $M_{E}$ be a manifold obtained by gluing two copies of $E$ together. If $G_{E}(x, y)$ is a Green's function obtained by compact exhaustion, then there is a constant $C$ such that for all $r>r_{0}>0$ and $x \in E \cap\left(M \backslash \overline{B_{p}(R)}\right)$ we have

$$
\begin{aligned}
& -G_{E}(p, x)+\sup _{\partial B_{p}\left(r_{0}\right)} G_{E}(p, \cdot) \\
& \quad \leq C\left(\int_{r_{0}}^{r(x)} \frac{t}{V_{p}(t)} d t+\sup _{\partial B_{p}\left(r_{0}\right)} G_{E}(p, \cdot)-\inf _{\partial B_{p}\left(2 r_{0}\right)} G_{E}(p, \cdot)\right) .
\end{aligned}
$$

(2) $M$ has a positive Green's function if and only if

$$
\int_{1}^{\infty} \frac{t}{V_{p}(t)} d t<\infty
$$

In particular, $E$ is nonparabolic if and only if $E$ is large. 
The following theorem can be considered as a generalization of the results in [20].

Theorem 6.4. Let $M$ be a complete noncompact manifold with nonnegative Ricci curvature outside a compact set. Suppose $b_{1}(M)$ is finite. Let $l$ be the number of large ends, and $s$ be the number of small ends. Then

$$
\mathscr{H}_{D}^{\infty}(M)=\mathscr{H}^{\infty}(M)
$$

and

$$
s+l=\operatorname{dim} \mathscr{H}^{0}(M)
$$

If we further assume that $l \geq 1$, then

$$
\mathscr{H}^{0}(M)=\mathscr{H}^{+}(M)
$$

and

$$
l=\operatorname{dim} \mathscr{H}_{D}^{\infty}(M)=\operatorname{dim} \mathscr{H}^{\infty}(M) .
$$

Here we let $\mathscr{H}^{0}(M)$ be the vector space spanned by those harmonic functions which are bounded on one side at each end with respect to some compact set, $\mathscr{H}^{+}(M)$ be the vector space spanned by the positive harmonic functions, $\mathscr{H}^{\infty}(M)$ be the space of bounded harmonic functions, and $\mathscr{H}_{D}^{\infty}(M)$ be the space of bounded harmonic functions with finite Dirichlet integral.

Proof. Theorem 6.3 asserts that an end is large if and only if it is nonparabolic. Using Lemma 6.1 and the fact that $M$ has nonnegative Ricci curvature outside a compact set, one can prove as in [20] that if $f$ is a positive harmonic function defined on an end $E_{r}$, then

$$
\sup _{\partial E_{2 r}} f \leq C \inf _{\partial E_{2 r}} f .
$$

From this Harnack-type inequality it follows that if $f$ is a positive harmonic function defined on $E$, then for any sequence $x_{i} \rightarrow \infty$ such that $x_{i} \in \partial E_{r\left(x_{i}\right)}$, we have

$$
\lim _{i \rightarrow \infty} f\left(x_{i}\right)=a
$$

for some $0 \leq a \leq \infty$. We claim that this is sufficient for showing that

$$
\lim _{\substack{x \rightarrow \infty \\ x \in E}} f(x)=a .
$$

In fact, let $x_{i} \rightarrow \infty$ be a sequence of points such that $x_{i} \notin \partial E_{r\left(x_{i}\right)}$. The fact that $E$ is a single end implies that there exists $R_{i} \rightarrow \infty$ such that $x_{i} \in E_{R_{i}}$. Without loss of generality, we may assume that $R_{i}$ be the largest value such that $x_{i} \in E_{R_{i}}$. Clearly, $x_{i}$ is contained in a bounded component of $E_{R_{i}} \backslash \partial E_{R_{i}}$. By the maximum principle, we obtain 


$$
\inf _{\partial E_{R_{i}}} f \leq f\left(x_{i}\right) \leq \sup _{E_{R_{i}}} f
$$

On the other hand, (6.1) asserts that both the upper bound and the lower bound tend to $a$. This confirms the claim. For $R>0$, let us define $\widetilde{B}_{p}(R)$ to be the union of $B_{p}(R)$ and the bounded components of $M \backslash \overline{B_{p}(R)}$. Let us also denote an unbounded components of $M \backslash \overline{B_{p}(R)}$ by $E_{R}$. Suppose $f$ is a bounded harmonic function on $M$. Then (see [20] and [24]) there exist $R_{i} \rightarrow \infty$ and harmonic functions $f_{i}$ on $\widetilde{B}_{p}\left(R_{i}\right)$ with $f_{i}$ being constant on $\overline{E_{R_{i}}} \cap \partial B_{p}\left(R_{i}\right)$ for each $E_{R_{i}}$, and $f=\lim _{i \rightarrow \infty} f_{i}$. It is easy to see [24] that $f_{i}$ has bounded Dirichlet integral. Hence the first equality of the theorem is true. Using the same argument as in [20] and [30], one can prove that if $E$ is an end, and $f$ and $g$ are positive harmonic functions on $E$ so that $f=g=0$ on $\partial E$, then $f$ is a multiple of $g$. Together with the results in [24], one can now proceed as in [20] and [30], and the theorem follows.

By the Harnack inequality in [14] and [28], it is easy to see that for a complete manifold which is quasi-isometric to a manifold satisfying the assumptions of Theorem 6.4 , the results of the theorem are still true.

\section{References}

[1] U. Abresch, Lower curvature bounds, Toponogov's theorem and bounded topology. I, Ann. Sci. Ecole Norm. Sup. 19 (1985) 651-670.

[2] U. Abresch \& D. Gromoll, Open manifolds with nonnegative Ricci curvature, J. Amer. Math. Soc. 3 (1990) 355-374.

[3] R. Bishop \& R. Crittenden, Geometry of manifolds, Academic Press, New York, 1964.

[4] M. Cai, Ends of Riemannian manifolds with nonnegative Ricci curvature outside a compact set, Bull. Amer. Math. Soc. 24 (1991) 371-377.

[5] M. Cai, T. H. Colding, \& D. Yang, A gap theorem for ends of complete manifolds, preprint.

[6] J. Cheeger \& D. Gromoll, The splitting theorem for manifolds of nonnegative Ricci curvature, J. Differential Geometry 6 (1971) 119-128.

[7] _ On the structure of complete manifolds of nonnegative curvature, Ann. of Math. (2) 92 (1972) 413-443.

[8] J. Cheeger, M. Gromov \& M. Taylor, Finite propagation speed, kernel estimates for functions of the Laplace operator, and the geometry complete Riemannian manifolds, J. Differential Geometry 17 (1982) 15-53.

[9] S. Y. Cheng, Finite dimensionality of the spaces of positive and bounded harmonic functions, preprint.

[10] S. Y. Cheng \& S. T. Yau, Differential equations on Riemannian manifolds and their geometric applications, Comm. Pure Appl. Math. 28 (1975) 333-354.

[11] H. Donnelly, Bounded harmonic functions and positive Ricci curvature, Math. Z. 191 (1986) 559-565.

[12] J-H. Eschenburg \& E. Heintze, An elementary proof of the Cheeger-Gromoll splitting theorem, Ann. Global Anal. Geom. 2 (1984) 249-260. 
[13] A. A. Grigor'yan, On the existence of positve fundamental solutions of the Laplace equation on Riemannian manifolds, Mat. Sb. 128 (3) (1985) 354-363; English transl. in Math. USSR-Sb. 56 (1987) 349-358.

[14] __ The heat equation on noncompact Riemannian manifolds, Mat. Sb. 182 (1) (1991) 55-87; English transl. in Math. USSR-Sb. 72 (1) (1992) 47-77.

[15] A. Kasue, A compactification of a manifold with asymptotically nonnegative curvature, Ann. Sci. Ecole Norm. Sup. 21 (1988) 593-622.

[16] _ Harmonic functions with growth conditions on a manifold of asymptotically nonnegative curvature I, Geometry and Analysis on Manifolds, Lecture Notes in Math., Vol. 1339, Springer, Berlin, 1988, 158-181.

[17] W. Lettman, G. Stampacchia \& H. F. Weinberger, Regular points for elliptic equations with discontinuous coefficients, Ann. Scuola Norm. Sup. Pisa. 17 (1960) 43-77.

[18] P. Li, Large time behavior of the heat equation on complete manifolds with non-negative Ricci curvature, Ann. of Math. (2) 124 (1986) 1-21.

[19] P. Li \& R. Schoen, $L^{p}$ and mean value properties of subharmonic functions on Riemannian manifolds, Acta Math. 153 (1984) 279-301.

[20] P. Li \& L. F. Tam, Positive harmonic functions on complete manfiolds with non-negative curvature outside a compact set, Ann. of Math. (2) 125 (1987) 171-207.

[21] _ Symmetric Green's functions on complete manifolds, Amer. J. Math. 109 (1987) 1129-1154.

[22] __ Complete surfaces with finite total curvature, J. Differential Geometry 33 (1991) 139-168.

[23] _ , The heat equation and harmonic maps of complete manifolds, Invent. Math. 105 (1991) 1-46.

[24] _ Harmonic functions and the structure of complete manifolds, J. Differential Geometry 35 (1992) 359-383.

[25] P. Li \& S. T. Yau, On the parabolic kernel of the Schrödinger operator, Acta Math. 156 (1986) 153-201.

[26] Z-D. Liu, Ball covering on manifolds with nonnegative Ricci curvature near infinity, preprint.

[27] B. Malgrange, Existence et approximation des solutions der équations aux dérivées partielles et des équations de convolution, Ann. Inst. Fourier (Grenoble) 6 (1955) 271355.

[28] L. Saloff-Coste, Uniformly elliptic operators on Riemannian manifolds, J. Differential Geometry 36 (1992) 417-450.

[29] C.-J. Sung, Harmonic functions under quasi-isometry, preprint.

[30] L.-F. Tam, Harmonic functions on connected sums of manifolds, Math. Z. 211 (1992) 315-322.

[31] N. Varopoulos, The Poisson kernel on positively curved manifolds, J. Functional Analysis 44 (1981) 359-380.

[32] __ Green's function on positively curved manifolds, J. Functional Analysis 45 (1982) 109-118.

[33] _ Green's function on positively curved manifolds. II, J. Functional Analysis 49 (1982) 170-176.

[34] _ _ Potential theory and diffusion on Riemannian manifolds, Conf. on Harmonic Analysis in Honor of Antoni Zygmund, Vols. I, II, Wadsworth Math. Ser., Wadsworth, Belmont, CA, 1983, 821-837.

[35] S. T. Yau, Harmonic functions on complete Riemannian manifolds, Comm. Pure Appl. Math. 28 (1975) 201-228. 